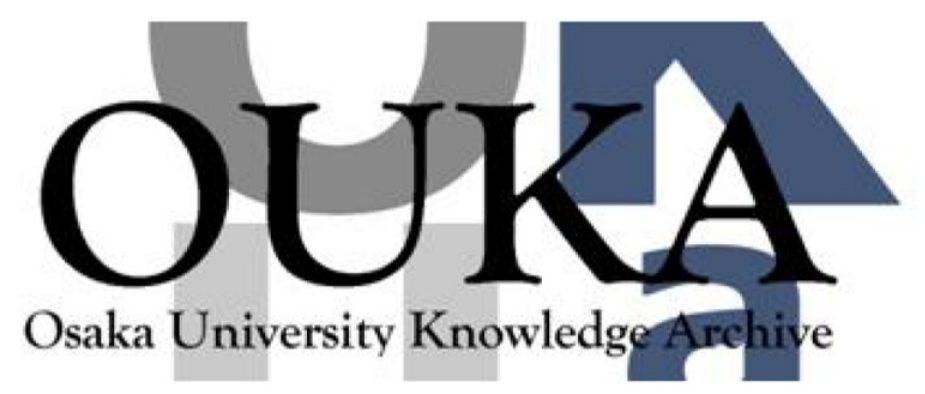

\begin{tabular}{|c|l|}
\hline Title & $\begin{array}{l}\text { Monte Carlo simulations of space-charge-limited } \\
\text { ion transport through collisional plasma } \\
\text { sheaths }\end{array}$ \\
\hline Author(s) & $\begin{array}{l}\text { Farouki, Rida T.; Hamaguchi, Satoshi; Dalvie, } \\
\text { Manoj }\end{array}$ \\
\hline Citation & Physical Review A. 44(4) p. 2664-p. 2681 \\
\hline Issue Date & $1991-08$ \\
\hline oaire:version & VoR \\
\hline URL & https://hdl.handle. net/11094/78521 \\
\hline rights & $\begin{array}{l}\text { Copyright (1991) by the American Physical } \\
\text { Society }\end{array}$ \\
\hline Note & \\
\hline
\end{tabular}

Osaka University Knowledge Archive : OUKA

https://ir. Library. osaka-u. ac. jp/

Osaka University 


\title{
Monte Carlo simulations of space-charge-limited ion transport through collisional plasma sheaths
}

\author{
Rida T. Farouki, Satoshi Hamaguchi, and Manoj Dalvie \\ IBM Thomas J. Watson Research Center, P. O. Box 218, Yorktown Heights, New York 10598 \\ (Received 11 February 1991; revised manuscript received 25 March 1991)
}

\begin{abstract}
Iterative Monte Carlo simulations are employed to study ion transport across the self-consistent electric field of a plasma sheath, under the influence of elastic collisions and charge-transfer reactions with ambient neutral species. Invoking arguments from kinetic theory, we express the retarding effect of encounters with neutral species as a "dynamical friction" force, proportional to the square of the mean ion velocity, whose coefficient $\kappa(z)$ is a function of position in the sheath through its dependence on the ion distribution $f(\mathbf{v}, z)$. We show that $\kappa(z)$ is determined by basic sheath parameters, such as the ion-to-neutral-species-mass ratio $m / M$, the mean free paths $\lambda_{e}$ and $\lambda_{c}$ for elastic scattering and charge transfer, and the ion distribution $f_{0}(\mathbf{v})=f(\mathbf{v}, 0)$ at the presheath-sheath boundary. When $m / M \geq 1$ or $\lambda_{c} \ll \lambda_{e}$, the Monte Carlo models indicate that $\kappa(z)$ is a relatively weak function, amenable to approximation by simple functional forms using parameters estimated from the simulations. By substituting such approximations into a simple continuum description-consisting of coupled ordinary differential equations for the electric field and mean ion velocity - substantively accurate models of the sheath structure are obtained at nominal computational expense, for regimes ranging from the nearly collisionless to the collision-dominated extremes.
\end{abstract}

\section{INTRODUCTION}

Low-pressure plasmas provide a means of achieving "anisotropic" or directional etching of thin films in integrated-circuit (IC) fabrication processes. The mechanism of this directional etching involves the continuous bombardment of a substrate by energetic positive ions that are accelerated while traversing the strong electric field of the plasma sheath. A collisionless sheath results in ions incident nearly normal to the surface, at energies comparable to the sheath potential, with only nominal deviations due to the relatively low ion temperature. The resulting narrow distribution of the ion incidence angles $\theta$ and energies $\varepsilon$,

$$
F(\theta, \varepsilon) \approx F_{0} \delta(\theta) \delta\left(\varepsilon-q V_{S}\right),
$$

where $q$ is the ion charge and $V_{S}$ is the sheath potential, gives excellent control over the etch anisotropy.

The operating pressures of process plasmas do not always guarantee a collisionless sheath, however. Collisions in the sheath induce a broadening of the distribution (1), reducing the directionality of the etch. Since higher pressures usually result in greater throughput, it is desirable to gain a detailed knowledge of the incident ion distributions that result from collisional sheaths. Such information can be useful in predicting the sectional profiles of surface features that evolve under ion bombardment (see, for example, Refs. [1]-[3]).

The incident ion distribution function is determined by the sheath structure (i.e., the variation of the electric field and the ion number density and average velocity in the sheath) and by the frequency and type of scattering events. Previous studies of ion transport across plasma sheaths have failed to incorporate models for the variation of the sheath electric field that are consistent with the ion motions and density distributions they induce $[2,4,5]$, or have relied on simple phenomenological models for the cumulative effects of ion-neutral-species scattering events $[6,7]$.

Our current goal is to present a detailed first-principles account of the role of collisional retardation of ions in influencing the self-consistent sheath structure, under the assumption of idealized models for the ion-neutralspecies interactions. Scattering events are assumed to be elastic (hard-sphere) collisions or charge-transfer reactions (i.e., the stripping of electrons from "cold" neutral species by fast ions to yield fast neutral species and slow ions), with cross sections that are independent of ion energy [8].

In Sec. II we discuss the ion transport problem in the context of kinetic theory. A “dynamical friction" term that arises on taking the first moment of the Boltzmann equation accounts for the mean rate of momentum loss due to ion-neutral-species collisions. Since this term involves a second-order moment of the ion distribution function $f(\mathbf{v}, z)$ in the sheath, Monte Carlo methods are invoked to assess its behavior and to identify regimes in which simple approximations are appropriate (see Secs. III and IV).

Although process plasmas are usually rf discharges, we shall concentrate on the dc transport problem in this paper. In the dynamics of $\mathrm{rf}$ sheaths, the ratio of the ion transit time to the rf period plays a key role, complicating the formulation of quantitative self-consistent sheath models (see, however, [9]). Nevertheless, an analysis of the dc transport problem is pertinent to rf sheaths in the limiting regimes where this ratio is small or large. In the former case, an ion experiences essentially a "single phase" of the rf cycle while traversing the sheath, and we may hope to model the transport problem "phase by phase." For the latter case, ions suffer many rf cycles 
during transit, and it is appropriate to model their transport in terms of an equivalent (i.e., time-averaged) dc sheath [7].

In terms of the plasma physics, we treat the sheath in isolation and make no attempt to solve the "complete plasma-sheath equation" (see [10]) governing the potential distribution for the entire discharge. The shortcoming of this approach lies in the introduction of certain ad hoc boundary conditions at the presheath-sheath interface, which must be regarded as adjustable "parameters" of our sheath models. Introducing these parameters allows us to focus on the ion transport problem within the sheath. Models of complete de discharges may be found in, for example, [11]-[13]. For sheath models that focus on the electron transport problem, including the effects of elastic and inelastic (excitation and ionization) collisions, and secondary electron emission from the cathode, see $[14,15]$.

Before proceeding to the kinetic theory and simulations, we briefly review some continuum models appropriate to limiting regimes. We consider onedimensional (planar) sheaths of negligible electron density [16]. Ions enter the sheath at $z=0$ from the presheath and are accelerated (between collisions with neutral species) by the sheath field, impinging on the cathode at $z=d$. A small ionization fraction is assumed, so that discrete Coulombic ion-ion encounters are ignorable and collisional heating of the neutral gas is insignificant. Furthermore, the thermal velocities of neutral species are taken to be negligible compared to ion velocities in the sheath, so that neutral species may be assumed at rest in collisions with ions. law

In the collisionless limit, the familiar Child-Langmuir

$$
E(z)=\left(\frac{6 m J^{2}}{q \epsilon_{0}^{2}}\right)^{1 / 3} z^{1 / 3}
$$

(where $q$ and $m$ are the ion charge and mass, $J$ is the constant ion current density, and $\epsilon_{0}$ is the permittivity of free space) is often quoted as a model for the electric-field profile in low-pressure plasma sheaths (e.g., [17]). It corresponds to a self-consistent solution of the collisionless ion equation of motion, the continuity equation, and Poisson's equation, under the assumption that the electric field $E(0)$ and ion velocity $u(0)$ at the presheath-sheath boundary are both zero [18].

A generalization of (2) to more realistic (nonzero) values for $E(0)$ and $u(0)$ is described in [19]. If $n_{e}$ and $T_{e}$ are the electron density and temperature for the plasma bulk, we have typically

$$
u(0)=u_{B}=\sqrt{k T_{e} / m},
$$

corresponding to the minimum incoming ion velocity (the Bohm velocity) for the formation of a stable sheath [20], and

$$
E(0)=\frac{k T_{e}}{2 e \lambda_{D}} \quad \text { where } \lambda_{D}=\left(\epsilon_{0} k T_{e} / e^{2} n_{e}\right)^{1 / 2},
$$

$E(0)$ being the magnitude of the field that "leaks" into the body of the plasma, i.e., is not subject to Debye damping ( $e$ is the magnitude of the charge on the electron, and $\lambda_{D}$ is the Debye length for the plasma bulk).

At higher pressures, a "collisional" version of the Child-Langmuir law has been cited in [17], based on the notion that, in a collision-dominated sheath, ions progress with a "drift" velocity $u(z)$ proportional to the local field: $u(z)=\mu E(z)$, where $\mu$ is the (constant) ion "mobility." When this relation is substituted into Poisson's equation by way of the continuity equation, it gives (see [17], p. 109):

$$
E(z)=\left[\frac{2 J}{\mu \epsilon_{0}}\right]^{1 / 2} z^{1 / 2}
$$

for the electric field [assuming again that $E(0)=0$ and $u(0)=0]$. In the context of plasma sheaths, however, constant ion mobility is not a credible assumption.

Constant-mobility models of charged particle transport are generally satisfactory for systems in which the assumption of a constant mean free time is appropriate. For elastic collisions with velocity-independent cross sections, this assumption is always valid at sufficiently low field strengths, such that thermal velocities dominate the field-induced drift velocity. For ions colliding with ambient neutral species of the same mass in a plasma sheath, however, a constant mean free path model is more appropriate for cross sections that are independent of ion velocity.

Conventionally, the constant-mobility approximation holds when the inequality

$$
\left[\frac{m}{M}+\frac{M}{m}\right] q E \lambda \ll k T_{i}
$$

is satisfied [21,22], where $m$ and $M$ are the ion and neutral-species masses, $\lambda$ is the mean free path for ionneutral-species collisions, $k$ is the Boltzmann constant, and $T_{i}$ is the ion "temperature" (i.e., when the kinetic energy acquired from the field over one mean free path is small compared to ion thermal energies). Note that $\lambda$ is inversely proportional to the neutral-species gas pressure $p_{g}$ at a fixed gas temperature. The criterion (6) is violated at the high field strengths and relatively low pressures characteristic of plasma sheaths; notwithstanding collisions with neutral species, the mean ion velocity $u(z)$ is better described as a "stream" velocity rather than a "drift" velocity.

In the collision-dominated regime, simple physical arguments suggest an equation for the mean ion motion of the form

$$
\frac{d u}{d t}=u \frac{d u}{d z}=\frac{q E}{m}-\kappa u^{2}
$$

the "drag" force $-\kappa u^{2}$ representing the retarding influence of ion-neutral-species encounters. Such an equation has been invoked in sheath models, for example, in [6] and [7]. The justification for Eq. (7) and its range of validity will be explored in detail below (where the nature of the coefficient $\kappa$ will also be elucidated).

For the present, we note that if $\kappa$ is constant and the 
field $E(z)$ is specified a priori, Eq. (7) can be integrated on multiplying by the integrating factor $\exp (2 \kappa z)$ to give $u^{2}(z)$ [and hence $u(z)$, since in the present context the stream velocity is non-negative] as an integral expression; see [6]. Moreover, it is clear that asymptotically $(z / \lambda \gg>1)$ the solution to (7) is approximately

$$
u(z)=\sqrt{q E(z) / m \kappa},
$$

which we identify as the "collision-dominated stream velocity." Note that Eq. (8) implies a field-dependent ion mobility, $\mu \propto E^{-1 / 2}$, emphasizing the erroneous nature of expression (5), which is based on the assumption of constant mobility.

We can also solve for a self-consistent electric field using the collision-dominated drift velocity (8), under the assumption that $E(0)=0$. This gives

$$
E(z)=\left(\frac{9 m \kappa J^{2}}{4 q \epsilon_{0}^{2}}\right)^{1 / 3} z^{2 / 3},
$$

a somewhat stronger power law than expression (5) for the case of constant $\mu$. Further discussion of ion mobility, including empirical evidence for the nonlinear dependence of mean velocities on the electric-field strength may be found in the literature; see [21]-[25] and references therein [26].

For sheaths of intermediate collisionality, however, the asymptotic relation (8) may not be attained, and the assumption $\kappa=$ const is questionable. The relation between $u(z)$ and $E(z)$ is then more involved, being defined by the solution to the simultaneous, nonlinear, first-order equations

$$
\frac{d E}{d z}=\frac{J}{\epsilon_{0} u}, \quad \frac{d u}{d z}=\frac{q E-m \kappa u^{2}}{m u}
$$

subject to the initial values (3) and (4). Here we use the constancy of the ion flux, $q n u=J$, to eliminate the ion number density $n$ from Poisson's equation $d E / d z$ $=q n / \epsilon_{0}$, giving the first equation above; the second is the momentum equation (7).

The coupled equations (10) may be regarded as defining a simple continuum model that is capable of yielding more accurate descriptions of the sheath structure at intermediate levels of collisionality $(1 \leq d / \lambda \leq 10$, say) than the models discussed above. The numerical integration of Eqs. (10) as an initial-value problem is a straightforward matter, even with a nonconstant "dynamical friction" coefficient $\kappa(z)$. By comparison with detailed Monte Carlo simulations we shall verify in Sec. IV that, in appropriate regimes, such integrations offer a good semiquantitative description of the sheath [27].

A precise definition of $\kappa(z)$ will be given in Sec. II, where we show that its variation is determined by the evolution of the ion velocity distribution with distance $z$ into the sheath. The simulation data described in Sec. IV indicate that when the ion-to-neutral-species mass ratio is not less than unity, or when charge-transfer reactions are dominant over elastic scattering in the ion-neutralspecies cross section, $\kappa(z)$ is a relatively weak function that is amenable to simple approximations.
Finally, we note that by setting $E=-d V / d z$ and integrating any of the three equations (2), (5), or (9) from $z=0$ to $d$, we obtain a corresponding expression relating the total potential drop $V_{S}$ across the sheath to the ion current density $J$ and the sheath thickness $d$. Such a relation also holds, implicitly, for the somewhat more sophisticated sheath model described by (10) — in general, we cannot vary all three of the macroscopic parameters $J$, $V_{S}$, and $d$ independently.

\section{KINETIC THEORY}

Before describing the Monte Carlo simulations (see Sec. III), it is instructive to examine the collisional sheath problem from the viewpoint of kinetic theory. We introduce the ion distribution function $f(\mathbf{v}, z)$, defined such that

$$
d n=f(\mathbf{v}, z) d \mathbf{v}
$$

represents the number density of ions at location $z$ whose velocity vectors lie within an element $d \mathbf{v}$ of velocity space, centered on $\mathbf{v}$. The total ion number density $n(z)$ at position $z$ is obtained by integrating (11) over all velocities, while the mean value $\langle\Phi\rangle$ of any function $\Phi(v)$ of ion velocity is obtained by multiplying (11) by $\Phi(\mathbf{v})$, integrating, and dividing by $n(z)$ (obviously, $\langle\Phi\rangle$ depends on $z$ in general).

Let $\mathbf{z}$ be a unit vector in the field direction. Since the system is axisymmetric about this direction, we may express the ion distribution function as

$$
f(\mathbf{v}, z)=f(v, \theta, z) \text { or } f\left(v_{z}, v_{t}, z\right)
$$

in terms of spherical or cylindrical coordinates in velocity space, where

$$
\begin{aligned}
& v=|\mathbf{v}|, \quad v_{z}=\mathbf{v} \cdot \mathbf{z}, \\
& \theta=\cos ^{-1} \frac{v_{z}}{v}, \quad v_{t}=\left(v^{2}-v_{z}^{2}\right)^{1 / 2} .
\end{aligned}
$$

The volume elements $d \mathbf{v}$ of velocity space corresponding to these coordinates are $2 \pi \sin \theta d \theta v^{2} d v$ and $2 \pi v_{t} d v_{t} d v_{z}$, respectively.

\section{A. Kinetic equations}

In the steady state, the ion distribution function $f(\mathbf{v}, z)$ in the sheath is governed by the time-independent Boltzmann equation

$$
v_{z} \frac{\partial f}{\partial z}+\frac{q E}{m} \frac{\partial f}{\partial v_{z}}=\left[\frac{\delta f}{\delta t}\right]_{\text {coll }},
$$

coupled with Poisson's equation

$$
\frac{d E}{d z}=\frac{q n}{\epsilon_{0}}
$$

where the ion number density $n$ at position $z$ is obtained by integrating (11) over velocity space:

$$
n(z)=\int f(\mathbf{v}, z) d \mathbf{v} \text {. }
$$


The "collision integral" on the right-hand side of (14) represents the net rate of change of the ion distribution function, at a given location $z$ in the sheath and point $\mathbf{v}$ of velocity space, due to two-body collisions with neutrals. For collisions between ions of mass $m$ and neutral species of mass $M$, with distribution functions $f(\mathbf{v}, z)$ and $F(\mathbf{V}, z)$, this integral is usually expressed [28] in the form

$$
\begin{aligned}
{\left[\frac{\delta f}{\delta t}\right]_{\text {coll }}=\int } & \int\left[f\left(\mathbf{v}^{\prime}\right) F\left(\mathbf{V}^{\prime}\right)-f(\mathbf{v}) F(\mathbf{V})\right] \\
& \times\left|\mathbf{v}^{\prime}-\mathbf{V}^{\prime}\right| \sigma(\psi, \varphi) d^{2} \Omega d \mathbf{V},
\end{aligned}
$$

where $\left(\mathbf{v}^{\prime}, \mathbf{V}^{\prime}\right)$ and $(\mathbf{v}, \mathbf{V})$ denote the velocities of an ion-neutral-species pair before and after a collision, and $\sigma(\psi, \varphi)$ is the corresponding differential cross section for scattering of the ion velocity, relative to that of the neutral species, by polar and azimuthal angles $\psi$ and $\varphi$, into the solid-angle element $d^{2} \Omega=\sin \psi d \psi d \varphi$ (in general, the differential cross section will also depend on the relative collision speed $\left.\left|\mathbf{v}^{\prime}-\mathbf{V}^{\prime}\right|\right)$. The total collision cross section $\sigma_{t}$ is obtained by integrating $\sigma(\psi, \varphi)$ over all angles:

$$
\sigma_{t}=\int \sigma(\psi, \varphi) d^{2} \Omega
$$

In (17), the precollision velocities $\left(\mathbf{v}^{\prime}, \mathbf{V}^{\prime}\right)$ and postcollision velocities $(\mathbf{v}, \mathbf{V})$ are related by a "scattering map"

$$
\left(\mathbf{v}^{\prime}, \mathbf{V}^{\prime}\right)=\mathcal{S}(\mathbf{v}, \mathbf{V})
$$

which depends on the collision impact parameter $b$, and possibly also on relative orientation angles, being determined by the details of the ion-neutral-species interaction. The differential cross section $\sigma(\psi, \varphi)$ is derived from (19).

We consider here only two idealized forms for the scattering map (19): elastic (hard-sphere) scattering, and "ion-stopping" charge-transfer reactions. Thus the differential cross section $\sigma(\psi, \varphi)$ is regarded as the sum of components $\sigma_{e}(\psi, \varphi)$ and $\sigma_{c}(\psi, \varphi)$ that describe these two forms of ion-neutral-species encounter. We denote the total cross sections for elastic scattering and charge transfer by $\sigma_{e}$ and $\sigma_{c}$, so that $\sigma_{t}=\sigma_{e}+\sigma_{c}$. Note that the magnitude of the ion velocity relative to the neutral species is conserved (i.e., $|\mathbf{v}-\mathbf{V}|=\left|\mathbf{v}^{\prime}-\mathbf{V}^{\prime}\right|$ ) for both these interactions.

From the scattering map (19) it is clear that the collision integral $(\delta f / \delta t)_{\text {coll }}$ is a function only of the ion velocity $\mathbf{v}$ and location $z$ within the sheath [for brevity, we suppress the $z$ dependence of the distribution functions in (17)]. This integral may be more conveniently expressed [29] as a difference between integrals over the velocity spaces $\mathbf{V}^{\prime}$ and $\mathbf{V}$ of neutral species:

$$
\begin{aligned}
{\left[\frac{\delta f}{\delta t}\right]_{\text {coll }}=} & \iint f\left(\mathbf{v}^{\prime}\right) F\left(\mathbf{V}^{\prime}\right)\left|\mathbf{v}^{\prime}-\mathbf{V}^{\prime}\right| \sigma(\psi, \varphi) d^{2} \Omega \frac{\partial\left(\mathbf{v}^{\prime}\right)}{\partial(\mathbf{v})} d \mathbf{V}^{\prime} \\
& -\iint f(\mathbf{v}) F(\mathbf{V})|\mathbf{v}-\mathbf{V}| \sigma(\psi, \varphi) d^{2} \Omega d \mathbf{V},(20)
\end{aligned}
$$

where we use the fact that $d \mathbf{v} d \mathbf{V}=d \mathbf{v}^{\prime} d \mathbf{V}^{\prime}$, and the Jacobian in the first integral has the form [29]

$$
\frac{\partial\left(\mathbf{v}^{\prime}\right)}{\partial(\mathbf{v})}=\left(\frac{\left|\mathbf{v}^{\prime}-\mathbf{v}^{\prime}\right|}{\left|\mathbf{v}-\mathbf{v}^{\prime}\right|}\right]^{3}
$$

We shall consider here only the case of spatially uniform cold neutral species, whose distribution function is taken to be

$$
F\left(\mathbf{V}^{\prime}, z\right)=n_{g} \delta\left(\mathbf{V}^{\prime}\right),
$$

$n_{g}$ being the (constant) number density of neutral species. Now (22) has been written in terms of the neutral velocity $\mathbf{V}^{\prime}$ before a collision; after a collision, of course, the neutral velocity $\mathbf{V}$ is not zero in general, and formally it is inconsistent also to write $F(\mathbf{V}, z)=n_{g} \delta(\mathbf{V})$ in the second term of (20). Our justification for doing so is based on the assumption of an extremely small ionization fraction, and a correspondingly small "hot" perturbation to (22) that represents the true equilibrium distribution of neutral species. [To be precise, $f(\mathbf{v}, z)$ and $F(\mathbf{V}, z)$ are actually governed by two individual Boltzmann equations, coupled through their collision integrals-assuming the form (22) for the neutral-species distribution allows us to dispense with one of these equations.]

Substituting (22) into (20) and integrating we obtain

$$
\left[\frac{\delta f}{\delta t}\right]_{\text {coll }}=n_{g} \sigma_{t} v\left[\int\left[\frac{v^{\prime}}{v}\right]^{4} f\left(\mathbf{v}^{\prime}\right) \frac{\sigma(\psi, \varphi)}{\sigma_{t}} d^{2} \Omega-f(\mathbf{v})\right] \text {, }
$$

where the ratio $v^{\prime} / v$, as a function of the scattering angles $(\psi, \varphi)$, is embodied in the symbolic expression (19). The system of equations (14)-(16), together with expression (23) for $(\delta f / \delta t)_{\text {coll }}$, define an initial-value problem for the ion distribution function, requiring a form

$$
f_{0}(\mathbf{v})=f(\mathbf{v}, 0)
$$

for $f(\mathbf{v}, z)$ at $z=0$ for its integration [30]. It should be noted, therefore, that the system of equations $(14)-(16)$ does not, without further information, determine the sheath thickness $d$.

In addition to the sheath thickness, the important macroscopic parameters are the ion current density $J$ and sheath potential $V_{S}$, given by

$$
J=q n(0) u(0), \quad V_{S}=\int_{0}^{d} E(\xi) d \xi,
$$

where $n(0) u(0)=\int v_{z} f_{0}(\mathbf{v}) d \mathbf{v}$ and the initial stream velocity $u(0)$ is assumed to have the Bohm value (3). As noted in Sec. I, the three quantities $J, V_{S}$, and $d$ are interrelated; only two of them may be freely chosen. If $d$ is specified a priori, the integration of Eqs. (14)-(16) is simply truncated at $z=d$. Otherwise, $d$ must be regarded as being specified implicitly through the expression (25) for $V_{S}$

\section{B. Scaling laws}

The system of integro-differential equations (14)-(16), together with expression (23), is difficult to solve in closed form, even under simplifying assumptions. In Secs. III and IV we describe indirect "solutions" by means of the Monte Carlo method. The goal of these simulations is to 
identify the principal physical effects governing the sheath structure, and to explore the range of validity of simpler models such as (10).

For the present, however, we can gain useful insight by casting Eqs. (14)-(16) and (23) in dimensionless form. We introduce the following transformations:

$\widehat{z}=\frac{z}{\lambda}, \quad \hat{\mathbf{v}}=\frac{\mathbf{v}}{v_{*}}, \quad \hat{f}=\frac{f}{f_{*}}, \quad \hat{E}=\frac{E}{E_{*}}, \hat{n}=\frac{n}{n_{0}}$,

where $\lambda=\left(n_{g} \sigma_{t}\right)^{-1}$ denotes the mean free path, $n_{0}=n(0)$ is the ion density at the presheath-sheath boundary, and the remaining scale values in (26) are given by

$$
v_{*}=\lambda \omega_{i}, \quad f_{*}=\frac{n_{0}}{v_{*}^{3}}, \quad E_{*}=\frac{\lambda q n_{0}}{\epsilon_{0}}
$$

$\omega_{i}=\left(q^{2} n_{0} / \epsilon_{0} m\right)^{1 / 2}$ being the ion plasma frequency. In terms of these dimensionless variables, Eqs. (14) and (15) may be written as

$$
\hat{v}_{z} \frac{\partial \hat{f}}{\partial \hat{z}}+\hat{E} \frac{\partial \hat{f}}{\partial \hat{v}_{z}}=\left[\frac{\delta f}{\delta t}\right]_{\text {coll }}^{\prime},
$$

the dimensionless form (denoted by the asterisk) of the collision integral on the right-hand side being equal to $\left(\lambda / v_{*} f_{*}\right)(\delta f / \delta t)_{\text {coll }}$, and

$$
\frac{d \hat{E}}{d \hat{z}}=\hat{n}
$$

Similarly, the dimensionless form of the normalization condition (16) is

$$
\hat{n}(\widehat{z})=\int \hat{f}(\widehat{\mathbf{v}}, \widehat{z}) d \widehat{\mathbf{v}} .
$$

The initial distribution (24) is also scaled accordingly: $\hat{f}_{0}=f_{0} / f_{*}$.

It is remarkable that the system of equations (28)-(30) exhibits no free parameters. Its solutions $\hat{f}, \hat{E}$, and $\hat{n}$ are therefore functions only of the dimensionless distance $\hat{z}=z / \lambda$ into the sheath and the initial distribution $\hat{f}_{0}$. The "collisionality" of the system is determined solely by the value $\hat{z}$ : the inequalities $\hat{z}<<1$ and $\hat{z}>1$ identify "collisionless" and "collision-dominated" regimes.

The parameter dependence of physically interesting quantities comes directly from the transformations (26). For example, the ion stream velocity $u=\left\langle v_{z}\right\rangle$ $=n^{-1} \int v_{z} f d \mathbf{v}$, and the electric field $E$ and corresponding potential $V=-\int_{0}^{z} E(\xi) d \xi$, may be expressed as

$$
\left\langle v_{z}\right\rangle=\lambda \omega_{i} g_{1}(\hat{z}), \quad E=\frac{\lambda q n_{0}}{\epsilon_{0}} g_{2}(\hat{z}), \quad V=\frac{\lambda^{2} q n_{0}}{\epsilon_{0}} g_{3}(\hat{z})
$$

while the average direction cosine for the ion motion, $\langle\cos \theta\rangle=n^{-1} \int\left(v_{z} / v\right) f d \mathbf{v}$, and the angular distribution, $p(\theta)=2 \pi \sin \theta n^{-1} \int f v^{2} d v$, have the form

$$
\langle\cos \theta\rangle=g_{4}(\hat{z}), \quad p(\theta)=g_{5}(\theta, \widehat{z}) .
$$

The dimensionless functions $g_{i}$ for $i=1, \ldots, 5$ also depend on the initial distribution function $\widehat{f}_{0}$, reflecting the dependence of sheath quantities on presheath parameters. The results of the numerical experiments (see Sec. IV) indicate that, for ion-to-neutral-species mass ratios not less than unity, $\langle\cos \theta\rangle$ and $p(\theta)$ approach a finite asymptotic value and a well-defined asymptotic function of $\theta$, respectively, as $\hat{z} \rightarrow \infty$.

It is interesting to observe how the quantities (31) and (32) scale with the gas pressure $p_{g}$. For a fixed gas temperature, the neutral density $n_{g}$ is proportional to $p_{g}$ and the ion density $n_{0}$ at the presheath-sheath boundary is also proportional to $p_{g}$ if we assume a constant ionization fraction. Thus $\lambda \propto 1 / p_{g}$ and $\omega_{i} \propto \sqrt{p_{g}}$, and for a fixed degree of collisionality (i.e., a given value of $\widehat{z}=z / \lambda$ ) we have

$$
\left\langle v_{z}\right\rangle \propto \sqrt{1 / p_{g}}, \quad V_{S} \propto \frac{1}{p_{g}},
$$

while $E,\langle\cos \theta\rangle$, and $p(\theta)$ do not depend on $p_{g}$. Conversely, if we vary the discharge parameters in such a manner that $p_{g} V_{S}=$ const, the degree of collisionality in the sheath, and hence the angular and energy distributions of ions incident on the cathode, will remain unchanged (assuming a fixed ionization fraction, electron temperature, initial ion distribution, etc.). These observations arise purely from a dimensional analysis of the collisional sheath problem, and thus apply equally to the simple continuum models of Sec. I and the detailed kinetic description defined by Eqs. (14)-(17); they are independent of the relation between $J, V_{S}$, and $d$ for the given sheath model.

\section{Dynamical friction}

As is well known, taking the integral of (14) over velocity space yields

$$
\frac{d}{d z}\left(n\left\langle v_{z}\right\rangle\right)=0
$$

expressing the constancy of ion flux. The left-hand side of (34) corresponds to the integral of the first term in (14). The fact that $f \rightarrow 0$ as $v_{z} \rightarrow \infty$ ensures that the integral of the second term in (14) will vanish, while the integral of the collision term $(\delta f / \delta t)_{\text {coll }}$ - which represents the time derivative of the ion density at a given position $z-$ is also zero (assuming a constant, steady-state ionization fraction).

We now take the first moment of the Boltzmann equation, multiplying both sides by $v_{z}$ and integrating over $\mathbf{v}$. The first term on the left can then be rewritten as

$$
\int v_{z}^{2} \frac{\partial f}{\partial z} d \mathbf{v}=\frac{d}{d z}\left(n\left\langle v_{z}^{2}\right\rangle\right)
$$

while the second term gives

$$
\frac{q E}{m} \int v_{z} \frac{\partial f}{\partial v_{z}} d \mathbf{v}=-\frac{q E}{m} n
$$

on integrating by parts. Thus we have the momentum equation 


$$
\frac{d}{d z}\left(n\left\langle v_{z}^{2}\right\rangle\right)=n\left(\frac{q E}{m}-R\right),
$$

where the quantity

$$
R(z)=-\frac{1}{n(z)} \int v_{z}\left(\frac{\delta f}{\delta t}\right)_{\text {coll }} d \mathbf{v}
$$

represents the dynamical friction force per unit ion mass at position $z$ [which was written as $\kappa u^{2}$ in Eq. (7), where $\left.u=\left\langle v_{z}\right\rangle\right]$.

Now the $z$ velocity of an ion may be expressed in the form $v_{z}=u+w_{z}$, where $w_{z}$ is the random component, relative to the stream velocity $u$, satisfying $\left\langle w_{z}\right\rangle=0$. Thus we have $\left\langle v_{z}^{2}\right\rangle=u^{2}+\left\langle w_{z}^{2}\right\rangle$, and making use of the fact that $n u=$ const, the left-hand side of (37) becomes

$$
\frac{d}{d z}\left(n\left\langle v_{z}^{2}\right\rangle\right)=n u \frac{d u}{d z}+\frac{d}{d z}\left(n\left\langle w_{z}^{2}\right\rangle\right) .
$$

If we interpret the second term on the right as the derivative of a "pressure" $P=n m\left\langle w_{z}^{2}\right\rangle$ in the $z$ direction, we see that the momentum equation corresponding to the first moment of (14) has the form

$$
u \frac{d u}{d z}=\frac{q E}{m}-R-\frac{1}{m n} \frac{d P}{d z} .
$$

Comparing (40) to the momentum equation (7) of our simple continuum model, we see that in the latter the pressure gradient term has been dropped, while the friction force $R$ has been expressed as $\kappa u^{2}$. We defer justification for neglecting the pressure gradient to the discussion of the Monte Carlo simulations, where it is verified empirically (in the case of ion-to-neutral-species mass ratios not less than unity, at least) that this term is of small magnitude compared to the other terms in (40). As is well known, it is impossible to generate a finite, closed set of continuum equations by taking successive moments of the Boltzmann equation-an ad hoc assumption must be invoked at some stage to ensure closure.

Consider now the dynamical friction force per unit mass, (38). By use of (23), this may be written as the difference $R^{+}-R^{-}$of the terms

$$
R^{+}=\frac{n_{g} \sigma_{t}}{n(z)} \int v_{z} v f(\mathbf{v}) d \mathbf{v}=\frac{\left\langle v v_{z}\right\rangle}{\lambda}
$$

and

$$
\begin{aligned}
R^{-} & =\left.\frac{n_{g}}{n(z)} \iint v_{z} v^{\prime} f\left(\mathbf{v}^{\prime}\right) \sigma(\psi, \varphi) d^{2} \Omega \frac{\partial\left(\mathbf{v}^{\prime}\right)}{\partial(\mathbf{v})}\right|_{\mathbf{v}^{\prime}=0} d \mathbf{v} \\
& =\frac{n_{g}}{n(z)} \iint v_{z} v^{\prime} f\left(\mathbf{v}^{\prime}\right) \sigma(\psi, \varphi) d^{2} \Omega d \mathbf{v}^{\prime}
\end{aligned}
$$

where in the second expression we have used the fact that $\left(v^{\prime} / v\right)^{3}=\partial\left(\mathbf{v}^{\prime}\right) /\left.\partial(\mathbf{v})\right|_{\mathbf{v}^{\prime}=0}$ to convert the integral for $R^{-}$ from the space $v$ to the space $v^{\prime}$.

Physically, the dynamical friction force $-m R$ exerted on an ion represents the mean loss of the $z$ component of its momentum per unit time, averaged over all possible collision configurations. This may be seen in the following manner.
Employing spherical polar coordinates in velocity space and writing $f\left(\mathbf{v}^{\prime}, z\right)=f\left(v^{\prime}, \theta^{\prime}, z\right)$ and $f(\mathbf{v}, z)$ $=f(v, \theta, z)$, we change the dummy integration variable $\mathbf{v}$ of (41) to $\mathrm{v}^{\prime}$ and substitute $v_{z}=v \cos \theta$ into (41) and (42) to obtain

$$
\begin{aligned}
-m R=\frac{n_{g}}{n(z)} \int & \int m\left(v \cos \theta-v^{\prime} \cos \theta^{\prime}\right) \\
& \times v^{\prime} f\left(v^{\prime}, \theta^{\prime}, z\right) \sigma(\psi, \varphi) d^{2} \Omega d \mathbf{v}^{\prime} .
\end{aligned}
$$

It should be noted in Eq. (43) that the expression $m\left(v \cos \theta-v^{\prime} \cos \theta^{\prime}\right)$ represents the $z$-momentum loss in a single collision, while $n_{g} v^{\prime} f\left(v^{\prime}, \theta^{\prime}, z\right) \sigma(\psi, \varphi) d^{2} \Omega / n(z)$ is the number of collisions per unit time that induce scattering into the solid-angle element $d^{2} \Omega$. These identifications substantiate the interpretation of $R$ given above.

Note that the term $R^{+}$in (41) depends only on the total cross section. Thus elastic scattering and charge transfer contribute to this term in proportion to their respective total cross sections, $\sigma_{e}$ and $\sigma_{c}$. However, the term $R^{-}$cannot be expressed merely in terms of total cross sections; a detailed specification of the relation between the velocities $v_{z}$ and $v^{\prime}$, in terms of the scattering angles $\psi$ and $\varphi$, is required to evaluate the integral (42).

Now for the idealized model of charge transfer in which ion-neutral-species encounters always yield ions essentially at rest, the charge-transfer contribution $R_{c}{ }^{-}$to (42) is obviously zero, since all ions satisfy $v_{z}=0$ after an encounter, regardless of their incident speed $v^{\prime}$. The elastic contribution $R_{e}^{-}$is less trivial, and we need to specify a definite form for $\sigma_{e}(\psi, \varphi)$ in order to compute it. For simplicity, we consider only the special case of hardsphere collisions, for which we have [31]:

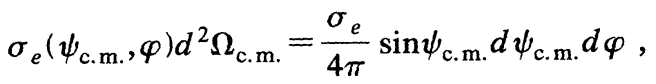

where $\psi_{\text {c.m. }}$ denotes the polar scattering angle measured in the center-of-mass frame, $\varphi$ denotes the azimuthal scattering angle about the $\mathbf{v}^{\prime}$ direction, and $\sigma_{e}=\pi a^{2}$ is the total elastic cross section ( $a$ being the sum of the radii of the ion and neutral species).

By straightforward trigonometric arguments, the polar angles $\theta^{\prime}$ and $\theta$ of the ion velocity with respect to the $z$ axis before and after a collision are related by

$$
\cos \theta=\cos \theta^{\prime} \cos \psi+\sin \theta^{\prime} \sin \psi \cos \varphi,
$$

where $\psi$ is the polar scattering angle measured in the laboratory frame. Denoting the ion and neutral-species masses by $m$ and $M$, we have the following relations [31]:

$$
\begin{aligned}
& \frac{v}{v^{\prime}}=\frac{\left(m^{2}+M^{2}+2 m M \cos \psi_{\mathrm{c} . \mathrm{m} .}\right)^{1 / 2}}{m+M}, \\
& \tan \psi=\frac{M \sin \psi_{\text {c.m. }}}{m+M \cos \psi_{\text {c.m. }}} .
\end{aligned}
$$

It is useful to note some important specializations of Eqs. (46). In the case of equal ion and neutral-species masses (i.e., $m=M$ ) we obtain 


$$
v=v^{\prime} \cos \frac{1}{2} \psi_{\text {c.m. } .}, \quad \psi=\frac{1}{2} \psi_{\text {c.m. }},
$$

whereas in both the limits $m<<M$ and $m \gg M$ we have $v=v^{\prime}, \quad$ and either $\quad \psi=\psi_{\text {c.m. }} \quad(m / M \rightarrow 0) \quad$ or $\quad \psi=0$ $(m / M \rightarrow \infty)$.

To simplify the expression for $R_{e}^{-}$, we make use of (45) and (46) and write the factor $v_{z}=v \cos \theta$ in (42) as

$$
\begin{aligned}
v_{z}= & \frac{\left(m^{2}+M^{2}+2 m M \cos \psi_{\mathrm{c} . \mathrm{m} .}\right)^{1 / 2}}{m+M} \\
& \times v^{\prime}\left(\cos \theta^{\prime} \cos \psi+\sin \theta^{\prime} \sin \psi \cos \varphi\right) .
\end{aligned}
$$

This is the only factor in the integrand of (42) that depends on the azimuthal scattering angle, and on averaging over $\varphi$ it is clear that the contribution of the term containing $\cos \varphi$ will vanish. Thus, rewriting the second expression in (46) as

$$
\cos \psi=\frac{m+M \cos \psi_{\text {c.m. }}}{\left(m^{2}+M^{2}+2 m M \cos \psi_{\text {c.m. }}\right)^{1 / 2}},
$$

and noting that $v^{\prime} \cos \theta^{\prime}=v_{z}^{\prime}$, we have

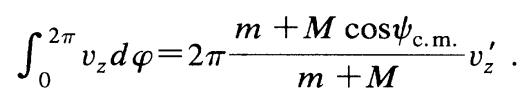

Making use of expressions (44) and (50) in Eq. (42), we now find that the integral for $R_{e}^{-}$may be written as

$$
\begin{aligned}
& R_{e}^{-}=n_{g} \sigma_{e} \int_{0}^{\pi} \frac{m+M \cos \psi_{\text {c.m. }}}{m+M} \frac{1}{2} \sin \psi_{\text {c. m. }} d \psi_{\text {c.m. }} \\
& \times \frac{1}{n(z)} \int v^{\prime} v_{z}^{\prime} f\left(\mathbf{v}^{\prime}\right) d \mathbf{v}^{\prime} .
\end{aligned}
$$

On evaluating the first integral above and writing $n\left\langle v v_{z}\right\rangle$ for the second, we obtain

$$
R_{e}^{-}=\frac{m}{m+M} n_{g} \sigma_{e}\left\langle v v_{z}\right\rangle .
$$

If we define mean free paths $\lambda_{e}=\left(n_{g} \sigma_{e}\right)^{-1}$ and $\lambda_{c}$ $=\left(n_{g} \sigma_{c}\right)^{-1}$ for elastic scattering and charge transfer, respectively, and note that

$$
\frac{1}{\lambda}=\frac{1}{\lambda_{e}}+\frac{1}{\lambda_{c}}
$$

we may then combine (52) with the result $R_{c}{ }^{-}=0$ and (41) to write the total dynamical friction force $R=R^{+}-R^{-}$ in the compact form

$$
R(z)=\left(\frac{M}{m+M} \frac{1}{\lambda_{e}}+\frac{1}{\lambda_{c}}\right)\left\langle v v_{z}\right\rangle .
$$

$R$ is seen to be proportional to the moment $\left\langle v v_{z}\right\rangle$ of the distribution function $f(\mathbf{v}, z)$, the coefficient of proportionality depending only on the ion-to-neutral-species mass ratio and the mean free paths for elastic scattering and charge transfer. Note that the factor $M /(m+M)$ in the elastic-scattering component of (54) decreases monotonically with the mass ratio $m / M$ from 1 when $m<<M$ to 0 when $m \gg M$, being equal to $\frac{1}{2}$ in the case $m=M$. The charge-transfer component, on the other hand, is independent of the mass ratio, and for a given distribution $f(\mathbf{v}, z)$ it always exerts a stronger retarding force per unit cross section than elastic scattering.

Identifying (54) with the dynamical friction term $\kappa u^{2}$ used in our continuum model (10), where $u=\left\langle v_{z}\right\rangle$, we now see that the coefficient $\kappa(z)$ may be defined precisely in terms of the ratio of moments of the ion distribution function $f(\mathbf{v}, z)$ :

$$
\kappa(z)=\left(\frac{M}{m+M} \frac{1}{\lambda_{e}}+\frac{1}{\lambda_{c}}\right) \frac{\left\langle v v_{z}\right\rangle}{\left\langle v_{z}\right\rangle^{2}} .
$$

We shall verify directly from the Monte Carlo simulations (see Secs. III and IV) that when the mass ratio $m / M$ is comparable to or greater than unity, the dimensionless ratio $\left\langle v v_{z}\right\rangle /\left\langle v_{z}\right\rangle^{2}$ is only weakly dependent on the distance $z$ into the sheath.

[Note that if the distribution function $f(\mathbf{v}, z)$ is such that $\left\langle v v_{z}\right\rangle \approx\langle v\rangle\left\langle v_{z}\right\rangle$ and $\left\langle v_{z}\right\rangle \approx\langle v\rangle\langle\cos \theta\rangle$ at each $z$, then the factor $\left\langle v v_{z}\right\rangle /\left\langle v_{z}\right\rangle^{2}$ in (55) is approximately $1 /\langle\cos \theta\rangle$. Physically it is reasonable to expect $\langle\cos \theta\rangle$, and any numerical factors incurred by these approximations, to approach asymptotic values over several mean free paths. This expectation is borne out by the simulation data.]

It is interesting to observe how certain well-known forms of space-charge-limited transport arise in limiting cases of the momentum equation (40). In the vacuum limit $\kappa \rightarrow 0$ (since $\left.\lambda_{e}, \lambda_{c} \rightarrow \infty\right)$, and we set $R=0$. Likewise $d P / d z=0$, since there are no collisions to induce an appreciable $z$ dispersion about the ion stream velocity. Thus, solving $u d u / d z \approx q E / m$ together with $d E / d z$ $=q n / \epsilon_{0}$ and $q n u=J$ gives the Child-Langmuir relation $J \propto d^{-2} V_{S}^{3 / 2}$, where $d$ is the sheath thickness and $V_{S}=\int_{0}^{d} E d z$ is the sheath voltage. In the opposite extreme, we neglect the inertia and pressure gradient terms in (40) and couple $q E / m \approx \kappa u^{2}$ (with $\kappa=$ const) to the Poisson and continuity equations, thereby obtaining the Forman relation [32] $J \propto d^{-5 / 2} V_{S}^{3 / 2}$ for high-pressure space-charge-limited current.

It should be noted that the dynamical friction term, based on the assumption of a constant collision frequency, has often been included in continuum models of ion and electron transport in discharges (see, for example, $[33,34])$.

\section{THE MONTE CARLO SCHEME}

The Monte Carlo method, based on integrating the independent trajectories of many ions subject to stochastic initial conditions and collision events during the sheath traversal, offers a practical means of "solving" Eqs. (14) - (16), together with (23) and (24), for the ion distribution $f(\mathbf{v}, z)$ and the self-consistent field $E(z)$.

The advantages of the Monte Carlo method lie in its directness (i.e., the absence of essential assumptions or approximations in describing the physics), and in the fact that the level of detail at which $f(\mathbf{v}, z)$ is resolved-as well as macroscopic variables dependent on it - is limited only by the number of particles followed. The main disadvantage is the computational expense, especially since the present context requires iteration on Monte Carlo runs to achieve self-consistency. Also, computa- 
tional efficiency favors simplified models of the ionneutral-species interaction.

\section{A. Integration of ion trajectories}

If the neutral species have a constant number density $n_{g}$ in the sheath, and the total ion-neutral-species cross section $\sigma_{t}$ is independent of ion velocity, the distribution of free paths $l$ between collisions will follow the exponential law

$$
P(l)=\frac{1}{\lambda} \exp (-l / \lambda)
$$

where $\lambda=\left(n_{g} \sigma_{t}\right)^{-1}$ is the mean free path for ionneutral-species collisions. Here $\sigma_{t}$ is assumed to consist of components $\sigma_{e}$ and $\sigma_{c}$ for elastic-scattering and charge-transfer reactions; no other forms of ion-neutralspecies interaction are considered at present. If $r$ is a random variable uniformly distributed on the unit interval,

$$
P(r)= \begin{cases}1 & \text { if } 0 \leq r \leq 1 \\ 0 & \text { otherwise }\end{cases}
$$

the distribution (56) may be generated [35] by applying the transformation

$$
l=-\lambda \ln r \text {. }
$$

The ion velocity component $v_{t}$ orthogonal to the field direction remains constant between collisions, while the (signed) component $v_{z}$ in the field direction increases monotonically with time. The total velocity $v$ is given by

$$
v=\left(v_{z}^{2}+v_{t}^{2}\right)^{1 / 2} \text {. }
$$

Since the (curvilinear) free path $l$ of an ion between collisions is the principal random parameter of the Monte Carlo simulation, it seems natural at first sight to formulate the equations of motion with respect to the intracollisional arc length $s$ of the ion trajectory as the independent variable:

$$
\frac{d v_{z}}{d s}=\frac{q E}{m v}, \frac{d z}{d s}=\frac{v_{z}}{v}, \frac{d t}{d s}=\frac{1}{v} .
$$

It will be noted, however, that these equations become singular as $v \rightarrow 0$. This problem may arise under the following circumstances: (i) in nearly head-on elastic collisions between ions and essentially stationary neutral species of equal mass, the ions lose most of their momentum; (ii) in charge-transfer encounters, ions strip electrons off thermal neutral species, transmuting them into slow ions; and (iii) if, due to multiple scatterings, ions arise in states with $v_{z}<0$ and $v_{t} \approx 0$, the acceleration by the sheath field causes points where $v \approx 0$ to occur along their trajectories.

Since truly stationary ions arise with vanishingly small probability, an adaptive step-selection procedure can, in principle, accommodate the numerical difficulties associated with the integration of Eqs. (60) for slow ions. In practice, however, such an approach was found in general to be less efficient and less robust than casting the equations of motion in the more familiar form

$$
\frac{d v_{z}}{d t}=\frac{q E}{m}, \quad \frac{d z}{d t}=v_{z}, \quad \frac{d s}{d t}=v,
$$

with time $t$ as the independent variable.

A fourth-order Runge-Kutta scheme with constant steps was used to integrate the equations (61) between collisions. As a nominal time step, we choose

$\Delta t=\frac{(d / \widetilde{v})}{N} \quad$ where $\widetilde{v}=\left[\min \left[1, \frac{\lambda}{d}\right) \frac{q V_{S}}{m}\right]^{1 / 2}$,

$d$ and $V_{S}$ being the sheath thickness and potential ( $\widetilde{v}$ is a "typical" ion velocity in the sheath). In Eq. (62), $N$ represents the approximate total number of steps required for an ion to traverse the sheath (the actual number of steps for each ion is, of course, influenced by the number and nature of the collisions it suffers). Typically, we have employed the rather conservative value $N=100$.

Before executing each step, an estimate of the corresponding increment in path length is made according to the second-order expansion

$$
\Delta s \approx v \Delta t+\frac{1}{2} \frac{v_{z}}{v} \frac{q E}{m}(\Delta t)^{2} .
$$

If $s$ is the cumulative path length since the last collision, and $s+\Delta s$ exceeds the current free path $l$, the time step is reduced accordingly [36] to the value

$$
\Delta t \approx \frac{v}{v_{z}} \frac{m v}{q E}\left[\left[1+2 \frac{v_{z}}{v} \frac{(l-s) q E}{m v^{2}}\right]^{1 / 2}-1\right],
$$

obtained by inverting (63), which brings the total path length (approximately) to the desired value $l$. The ion trajectory is complete after this step, and a new collision must be invoked.

Small fractional discrepancies between the nominal and computed free paths of ions arise in this scheme, due to the approximate formula (64) for choosing the final time step. These errors can be eliminated entirely by reverting to Eqs. (60) - assuming the ion is not nearly at rest - on the last step, but this measure was unnecessary in practice, since the errors were determined empirically to be quite insignificant and nonsystematic.

\section{B. Treatment of collisions}

A collision is invoked at the end of each computed ion free path $l$. The collision is randomly determined to be an elastic-scattering or a charge-transfer event according to the probabilities $P_{e}=\sigma_{e} / \sigma_{t}$ and $P_{c}=\sigma_{c} / \sigma_{t}$.

In a charge-transfer encounter between an ion and a neutral species, the net effect is to exchange the "identities" of these particles: a fast ion strips an electron from a thermal neutral, producing a fast neutral species and a thermal ion. Assuming a small ionization fraction, the fast neutral species represent only a minor perturbation of the ambient gas and we subsequently ignore them. Before commencing on its next free path, the "new" ion is assigned a random velocity vector selected from a Maxwellian distribution characterized by the neutral gas temperature.

Elastic-scattering events are modeled on the collisions 
of hard spheres. If $b$ is the collision impact parameter, we introduce the dimensionless quantity $\beta=b / a$ (where $a$ represents the sum of the ion and neutral-species radii: $\sigma_{e}=\pi a^{2}$ ). The distribution of impact parameters is then described by

$$
P(\beta)=\left\{\begin{array}{l}
2 \beta \text { if } 0 \leq \beta \leq 1 \\
0 \text { otherwise } .
\end{array}\right.
$$

The distribution (65) may be generated from the uniform random distribution (57) by invoking the transformation

$$
\beta=\sqrt{r} \text {. }
$$

The neutral species is assumed stationary on impact, while the ion velocities before and after the collision are denoted by $\mathbf{v}^{\prime}$ and $\mathbf{v}$. Relative to its incoming velocity, the ion is scattered by a polar angle

$$
\psi=\cos ^{-1} \frac{2 \beta^{2}+(M-1)}{\left[4 M \beta^{2}+(M-1)^{2}\right]^{1 / 2}},
$$

where $M=m / M$ is the ion-to-neutral mass ratio. When $M<1$, the scattering angle (67) decreases monotonically from $\psi=\pi$ to $\pi$ to $\psi=0$ as $\beta$ increases from 0 to 1 ; backscattering $\left(\psi>\frac{1}{2} \pi\right)$ occurs for impact parameters satisfying

$$
\beta<\sqrt{\frac{1}{2}(1-M)} .
$$

For $M>1$ there is no backscatter, the angle (67) increasing from $\psi=0$ at $\beta=0$, attaining the extremum value

$$
\psi_{\max }=\cos ^{-1} \frac{\left(M^{2}-1\right)^{1 / 2}}{M} \text { at } \beta=\left(\frac{M-1}{2 M}\right)^{1 / 2} \text {, }
$$

and then decreasing to $\psi=0$ at $\beta=1$. Finally, when $M=1, \psi=\cos ^{-1} \beta$ and the scattering angle decreases monotonically from $\psi=\frac{1}{2} \pi$ at $\beta=0$ to $\psi=0$ at $\beta=1$. Although there is no backscatter in this case, a sequence of collisions may nevertheless cause ions to acquire negative velocities in the field direction.

The azimuthal scattering angle $\varphi$ about the incoming ion velocity $\mathbf{v}^{\prime}$ is assumed to be random, with a uniform distribution in the range $0 \leq \varphi<2 \pi$. The ion speeds $v^{\prime}$ and $v$ before and after scattering are related by

$$
\frac{v}{v^{\prime}}=\frac{\left[4 M \beta^{2}+(M-1)^{2}\right]^{1 / 2}}{M+1} .
$$

Note that, for all $M$, the ratio $v / v^{\prime}$ increases monotonically from $|M-1| /(M+1)$ at $\beta=0$ to unity at $\beta=1$. Thus $v=0$ when $\beta=0$ and $M=1$ (i.e., head-on collisions between ions and stationary neutral species of equal mass leave the ions at rest).

After each scattering event, it is necessary to compute the components $v_{z}$ and $v_{t}$ of the ion velocity $\mathbf{v}$ to serve as initial values for the next free path. In terms of the polar angle $\theta^{\prime}$ of the incoming ion and the scattering angles $(\psi, \varphi)$ we have

$$
\begin{aligned}
& v_{z}=v\left(\cos \theta^{\prime} \cos \psi+\sin \theta^{\prime} \sin \psi \cos \varphi\right), \\
& v_{t}=\left(v^{2}-v_{z}^{2}\right)^{1 / 2},
\end{aligned}
$$

on making use of expression (45) for the direction cosine after a collision.

\section{Sampling techniques}

In the Monte Carlo simulations, the stream velocity $u(z)=\left\langle v_{z}\right\rangle$ at position $z$ is obtained by sampling the velocities $v_{z}$ of individual ions in the field direction at equal time intervals and averaging over all ions. Other moments of the ion distribution function of interest are obtained in a similar manner.

For this purpose, the sheath domain $z \in[0, d]$ is discretized into $N_{z}$ contiguous elements of width $\Delta z=d / N_{z}$. Since variations in the sheath profiles over length scales comparable to the mean free path $\lambda$ can be expected, $N_{z}$ must be chosen such that $\Delta z / \lambda$ is small compared to unity. At each sampling time, an ion contributes to the average velocity $u_{i}$ corresponding to the element $i$ in which it is located (the value $u_{i}$ is to be identified with the midpoint $z_{i}=\left(i-\frac{1}{2}\right) \Delta z$ of the $i$ th element $)$.

In addition to the stream velocity, other averages are computed, such as the mean direction $\operatorname{cosine}\langle\cos \theta\rangle$ of the ion motions, the quantity $\left\langle v v_{z}\right\rangle$ which appears in the dynamical friction coefficient (55), the mean-square transverse velocity $\left\langle v_{t}^{2}\right\rangle$, and the variance $\left\langle w_{z}^{2}\right\rangle=\left\langle\left(v_{z}-u\right)^{2}\right\rangle$ of $v_{z}$, which may be determined once the profile for $u(z)$ converges, and is of interest in assessing the relative importance of the pressure gradient term in (40).

The discrete velocity values $\left\{u_{i}\right\}$ give rise to discrete number densities

$$
n_{i}=\frac{J}{q u_{i}} \text { for } i=1, \ldots, N_{z}
$$

through the continuity equation, where $J$ is the (constant) ion current density. The sheath electric field is then obtained by integrating $d E / d z=q n(z) / \epsilon_{0}$ :

$$
E(z)=E_{0}+\frac{q}{\epsilon_{0}} \int_{0}^{z} n(\xi) d \xi
$$

Here, $E_{0}=E(0)$ denotes the magnitude of the electric field at the presheath-sheath boundary, given by (4). Since only the discrete values (72) are available for $n(z)$, we compute discrete values $E_{i}$ of (73) at the points $z_{i}$ by numerical quadrature.

A number of options are available for interpolating between the discrete values $E_{i}, u_{i}$, and $n_{i}$ which characterize the sheath structure in the simulations. When the total number of ions is large $\left(10^{4}-10^{6}\right)$ and sufficient elements $\left(N_{z} \approx 50\right.$, say) are used in discretizing the sheath domain, it has been found that higher-order schemes (e.g., cubic splines) offer little advantage, in terms of speed of convergence or smoothness of the final profiles, over the simplest (i.e., piecewise-linear) approach.

\section{Iteration to a self-consistent solution}

A self-consistent sheath model is realized by iterating the Monte Carlo simulations. This iteration begins by assuming an initial profile $E_{0}(z)$ for the electric field. Ion trajectories, punctuated by collision events, are then com- 
puted in this field and the resulting velocity profile $u_{0}(z)$ is determined by the sampling procedure described above. Equations (72) and (73) are then used to generate a new profile $E_{1}(z)$ for the field, with which a new Monte Carlo simulation may be performed.

This process is repeated until the sequence of field profiles $E_{0}(z), E_{1}(z), E_{2}(z), \ldots$ converges to some prescribed tolerance, at which point the ion motions and density distributions - including the retarding effects of collisions-will self-consistently generate the electric field profile that induces them. The fractional change in the field profile at the end of iteration $i$ is taken to be

$$
\epsilon_{i}=\frac{\int_{0}^{d}\left|E_{i}(z)-E_{i-1}(z)\right| d z}{\int_{0}^{d}\left|E_{i-1}(z)\right| d z},
$$

and a similar error measure can be defined for the velocity profile. Typically, no more than three or four iterations were necessary to achieve convergence of the electric-field profile to a fraction of $1 \%$ using approximately $10^{4}$ ions. The converged result was also found to be entirely independent of the adopted initial field profile $E_{0}(z)$, provided the corresponding potential drop $V_{S}$ across the sheath was reasonably commensurate with the equilibrium value. [We have not been motivated to attempt a convergence analysis for our method, being content with the empirical observation that manipulating $E_{0}(z)$ would influence the speed but not the fact of convergence when the initial and final values of $V_{S}$ do not differ by a large factor.]

The initial velocity components $\left(v_{z}, v_{t}\right)$ of ions entering the sheath are selected from the normalized distribution

$$
\begin{aligned}
P\left(v_{z}, v_{t}\right)= & 2 \pi v_{t}\left[\frac{3}{2 \pi \alpha^{2} u_{B}^{2}}\right]^{3 / 2} \\
& \times \exp \left[-\frac{3}{2} \frac{\left(v_{z}-u_{B}\right)^{2}+v_{t}^{2}}{\alpha^{2} u_{B}^{2}}\right],
\end{aligned}
$$

which corresponds to an isotropic Maxwellian distribution superposed on the initial stream velocity $u(0)=u_{B}$ in the $z$ direction [note in (75) that $-\infty<v_{z}<+\infty$ and $\left.0 \leq v_{t}<+\infty\right]$. The root-mean-square random velocity associated with the Maxwellian component of (75) is a fraction $\alpha$ of the directed Bohm velocity $u_{B}$, corresponding to an ion temperature at $z=0$ given by $T_{i}=m \alpha^{2} u_{B}^{2} / 3 k$.

The normal distributions in (75) cannot be generated by a simple mapping of just a single uniformly distributed random variable - the required transformation involves the inverse error function $\operatorname{erf}^{-1}(r)$. However, several efficient alternatives for sampling from normal distributions are available (see [37], pp. 117-127); we have relied on a simple "rejection" method.

Expression (75) corresponds to our choice for the initial form (24) of the ion distribution function. The assumption of an isotropic Maxwellian component is arbitrary, intended to account for the influence of collisions in the acceleration of ions to the Bohm velocity prior to entering the sheath [note that in reality we might expect the dispersion of $v_{z}$ about $u_{B}$ to differ in general from those of the Cartesian components $\left(v_{x}, v_{y}\right)$ of $v_{t}$ about 0$]$.

\section{RESULTS OF THE SIMULATIONS}

We now describe representative results from the simulations, and assess the simple continuum model (10) against them. The primary dimensionless parameters that determine the sheath structure are the ion-toneutral-species mass ratio $m / M$; the relative magnitudes of the total cross sections for elastic scattering and charge transfer, $\sigma_{e}$ and $\sigma_{c}$; the value $\alpha$ that measures the random component of the initial ion distribution function; and the sheath thickness measured in mean free paths, $d / \lambda$.

Note that the mass ratio is unimportant when charge transfer is the dominant mode of ion-neutralspecies interaction $\left(\sigma_{c} \gg \sigma_{e}\right)$. Also, as emphasized in Sec. II, the sheath thickness $d$ does not enter explicitly into the sheath structure other than as a point of truncation in the integration of the sheath equations. Thus we need only model the sheath as far as is required for a well-defined asymptotic behavior to ensue, typically no more than several mean free paths [38].

A number of cases, employing $10^{6}$ ions each, were iterated until they converged to a fractional error of 0.005 or less in the electric field. The parameters defining these runs are enumerated in Table I. Run A3 may be regarded as a "reference" against which the results of other runs are to be compared. (The nominal physical model for this run is the time-averaged sheath of a $\mathrm{rf}$ discharge in $\mathrm{O}_{2}$, with assumed neutral-species gas temperature $T_{g}=350 \mathrm{~K}$, electron temperature $T_{e}=10^{5} \mathrm{~K}$, ionization fraction $10^{-4}$, sheath thickness $d=2 \mathrm{~mm}$, and total ion-neutral-species cross section $\sigma_{t}=150 \AA^{2}$. The pressure $p_{g}=61.3 \mathrm{~m}$ Torr gives $d / \lambda=5$ in this case; $p_{g}$ is varied to adjust $d / \lambda$ in other runs.) The value of $\alpha$ for each run was chosen in an ad hoc manner, attempting to account for the importance of collisions in the presheath region.

The first five runs (sequence A) illustrate the importance of the mass ratio $m / M$ for the case of elastic scattering only and a fixed degree of collisionality. In the next three runs (sequence $B$ ), we examine the significance of the ratio $\sigma_{e}: \sigma_{c}$ of the cross sections for elastic scattering and charge exchange, for a fixed mass ratio $(m / M=1)$ and degree of collisionality (note that B3 is identical to A3, being repeated in Table I only for notational convenience). Sequence $\mathrm{C}$ is intended to illustrate the influence of the parameter $d / \lambda$ on the angular and energy distributions of incident ions.

Figure 1 illustrates in dimensionless form the converged profiles for the electric field, the ion stream and random velocities, and the ion number density in run A3 [with $E$ scaled by the mean field $E_{m}=V_{S} / d, u$ by the maximum velocity $u_{m}=\left(u_{B}^{2}+2 q V_{S} / m\right)^{1 / 2}$ in the absence of collisions, and $n$ by the initial value $n_{0}=n(0)$.

\section{A. Momentum balance}

In Eq. (40) we take the dynamical friction and pressure gradient terms to the left-hand side, and divide by the lo- 
TABLE I. Parameters for the Monte Carlo simulations.

\begin{tabular}{ccccc}
\hline run & $m / M$ & $\sigma_{e}: \sigma_{c}$ & $d / \lambda$ & $\alpha$ \\
\hline A1 & 0.25 & $1: 0$ & 5 & 0.5000 \\
A2 & 0.50 & $1: 0$ & 5 & 0.3750 \\
A3 & 1.00 & $1: 0$ & 5 & 0.2500 \\
A4 & 2.00 & $1: 0$ & 5 & 0.1875 \\
A5 & 4.00 & $1: 0$ & 5 & 0.1000 \\
B1 & 1.00 & $0: 1$ & 5 & 0.1000 \\
B2 & 1.00 & $1: 1$ & 5 & 0.1750 \\
B3 & 1.00 & $1: 0$ & 5 & 0.2500 \\
C1 & 1.00 & $1: 0$ & 1 & 0.2500 \\
C2 & 1.00 & $1: 0$ & 2 & 0.2500 \\
C3 & 1.00 & $1: 0$ & 4 & 0.2500 \\
\hline \hline
\end{tabular}

cal acceleration due to the electric field:

$$
\frac{m u}{q E} \frac{d u}{d z}+\frac{m R}{q E}+\frac{1}{q E n} \frac{d P}{d z}=1
$$

Note that in the present context, the three terms in (76) are all nominally non-negative. We can ascertain their relative importance, as a function of distance into the sheath, directly from the simulations.

These terms are to be interpreted as follows: the first represents the fraction of the force on ions due to the electric field that actually goes into accelerating them to their mean (stream) velocity $u$; the second indicates how much of this force is expended in overcoming the mean momentum loss per unit time of ions due to collisions with neutral species (dynamical friction); and the third accounts for the need to overcome the backward transport of momentum due to an increasing dispersion of the ion velocities about the mean $u$ with distance $z$ into the sheath (also a direct consequence of ion-neutral-species collisions).

Figure 2 illustrates the measured behavior of these terms for three runs from sequence $A$. Note that computing the "inertia" and pressure gradient terms in (76) requires a numerical differentiation of the profiles for $u$ and $n\left\langle w_{z}^{2}\right\rangle$, a process that inherently magnifies any noise in these profiles. Nevertheless, in all runs the sum of the three terms in (76) was found to be within a few percent of unity over the entire sheath extent once the profiles had converged to a self-consistent solution.

Some common features of the behavior of the three terms in (76) are noteworthy in the runs of sequence $A$. Initially, the inertia term dominates, indicating that the work done by the electric field goes into the directed kinetic energy of the ion stream velocity. As the latter increases, however, collisions with neutral species dissipate an increasing proportion of the directed motion, as indicted by the rise of the second term in (76) and the decay of the first. A quasiequilibrium state is attained, in which the electric field acts principally to overcome dynamical friction, and the ion stream velocity exhibits an appreciable increase only over many mean free paths. The extent of the transient region required to establish this "collision-dominated" regime increases monotonically with the mass ratio $m / M$.

For mass ratios greater than or equal to unity, the
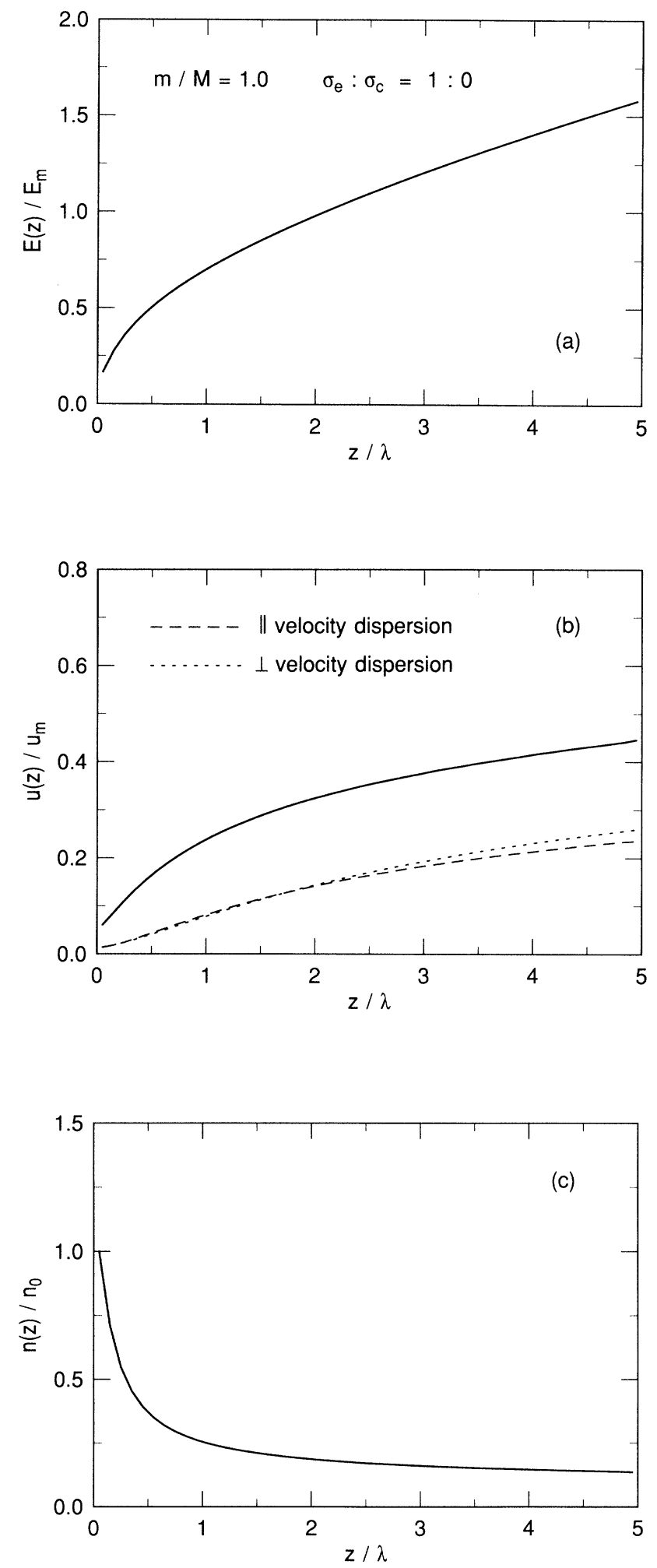

FIG. 1. Profiles for the converged self-consistent sheath structure in run A3: (a) the electric field; (b) the ion stream velocity and the longitudinal and transverse velocity dispersions; and (c) the ion number density. 
"pressure gradient" term in (76) was found to be relatively insignificant over the entire extent of the sheath, justifying the neglect of this term in formulating the simple continuum model (10) for collisional sheaths. When the ions are less massive than the neutral species, however, their longitudinal and transverse velocity dispersions be-
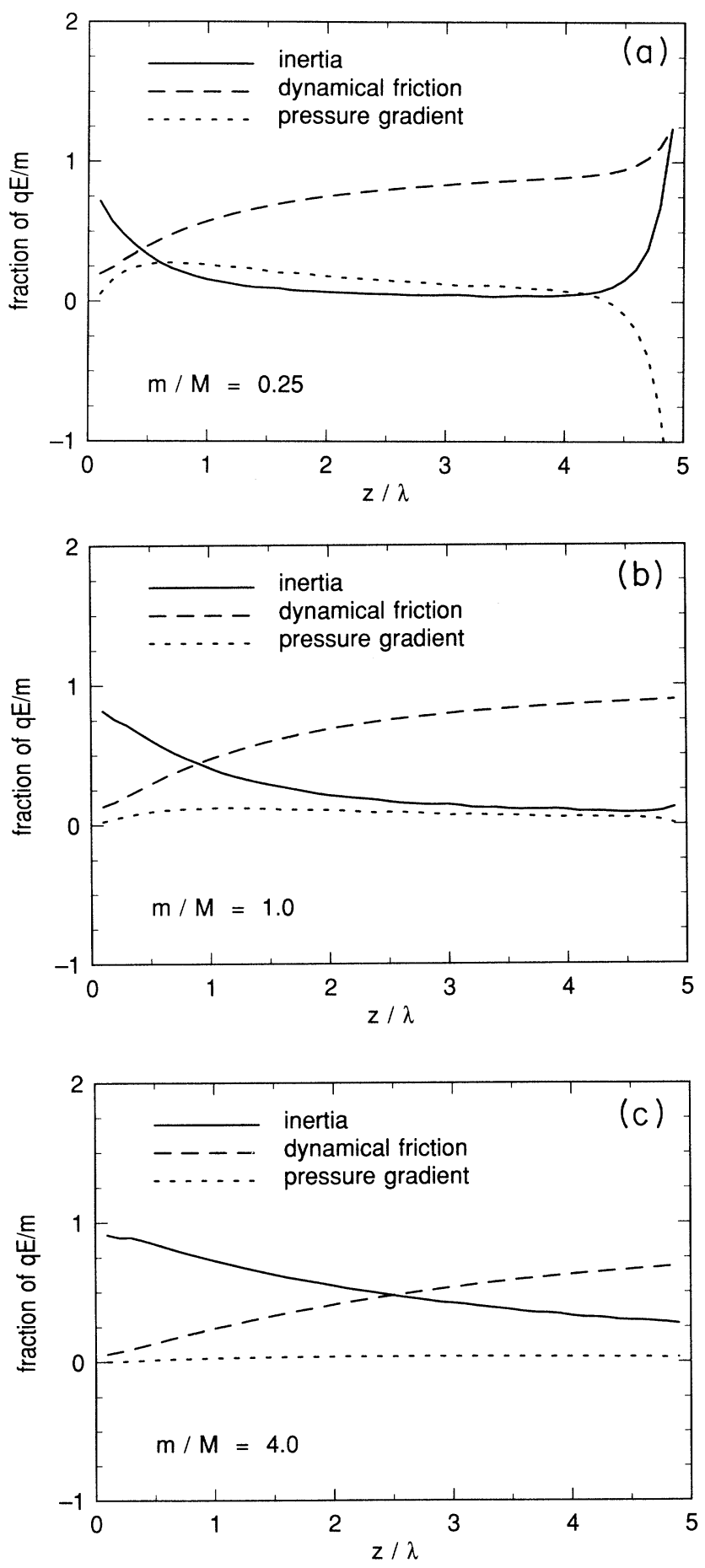

FIG. 2. The three terms of the momentum-balance equation (76) for the case of elastic scattering with $d / \lambda=5$ and mass ratios $m / M=0.25,1.0$, and 4.0 (runs A1, A3, and A5). come comparable to the stream velocity, and the presence of an absorbing wall at $z=d$ induces a significant perturbation in their distribution function within the last mean free path. As illustrated by the case $m / M=0.25$ in Fig. 2, this perturbation manifests itself in the momentum balance equation through the development of a strong negative pressure gradient and a sudden final acceleration of the ions near the cathode.

If the charge-transfer cross section is comparable to or greater than the elastic-scattering cross section, the behavior of the three terms in (76) resembles the high mass-ratio runs of sequence A; Fig. 3 illustrates the momentum balance for run B1. Note that the ions will have a negligible transverse velocity dispersion throughout the sheath when charge transfer dominates and the parameter $\alpha$ of the initial distribution (75) is small compared to unity.

Lawler [39] presents solutions of the Boltzmann equation for a dc sheath (or "cathode fall"), for the case of charge transfer only, with an assumed constant or linearly increasing electric field $E(z)$ and a plane ion source at $z=0$ or a uniform production rate for ions over $0 \leq z \leq d$ (see also [14]). From these solutions an "equilibration" distance for the ions can be estimated, over which they attain $90 \%$ of their equilibrium drift velocity. For the case most nearly resembling our self-consistent simulations - a plane source and a linear field - this distance is roughly $1.7 \lambda$, in excellent agreement with the behavior seen in Fig. 3 if we interpret the equilibration distance as that required for the inertia term on the lefthand side of Eq. (76) to drop below $10 \%$ of the total.

\section{B. The dynamical friction coefficient $\kappa(z)$}

Recall that the definition (55) of the dynamical friction coefficient $\kappa(z)$ involves the ratio of the moments $\left\langle v v_{z}\right\rangle$ and $\left\langle v_{z}\right\rangle^{2}$ of the ion distribution function (see Sec. II). Since the Monte Carlo simulations permit a direct measurement of these moments as functions of position $z$ in the sheath, we can ascertain the variation of $\kappa(z)$ and for-

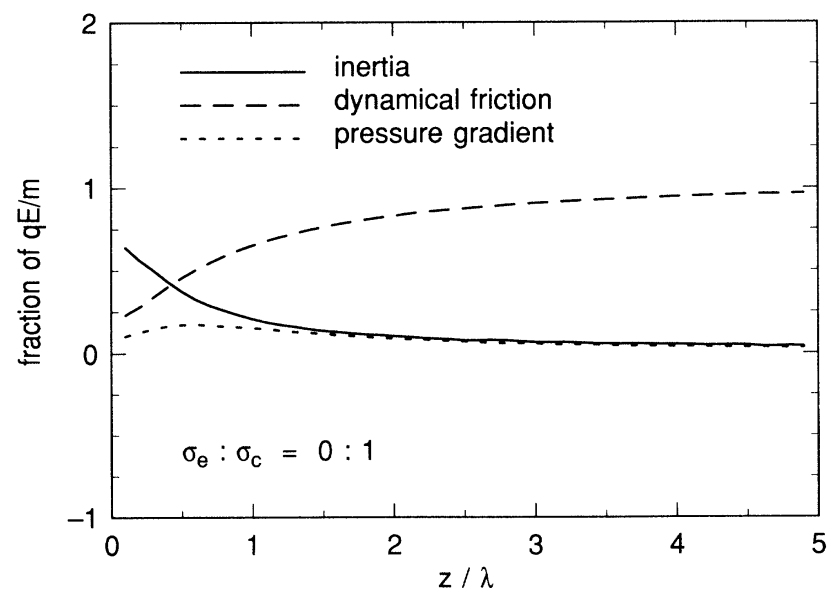

FIG. 3. The terms of the momentum-balance equation (76) for the case of charge transfer with $d / \lambda=5$ (run B1). 
mulate simple empirical functional forms for it.

Figure 4 illustrates the measured behavior of $\left\langle v v_{z}\right\rangle /\left\langle v_{z}\right\rangle^{2}$ for the five runs of sequence $\mathbf{A}$. It is evident that when $m / M \geq 1$, this ratio exhibits a mild monotonic increase to an asymptotic value between about 1.5 (when $m / M=1$ ) and 1.0 (when $m / M \rightarrow \infty$ ). When $m / M<1$, however, we see that $\left\langle v v_{z}\right\rangle /\left\langle v_{z}\right\rangle^{2}$ may attain large $(\geq 2.0)$ intermediate values, and furthermore is not monotone increasing; the perturbation of the ion distribution function near $z=d$ due to the absorbing wall induces a sudden decline within the last mean free path.

For a fixed mass ratio, we find that the variation of $\left\langle v v_{z}\right\rangle /\left\langle v_{z}\right\rangle^{2}$ is not strongly dependent on the relative sizes of the elastic-scattering and charge-transfer cross sections-Fig. 5 shows results for the three runs of sequence B. For mass ratio $m / M=1$ and a fixed total cross section $\sigma_{t}$, a modest systematic increase in the dynamical friction coefficient is observed as we proceed from the case of pure elastic scattering to that of pure charge transfer. When charge transfer is the dominant component of $\sigma_{t}$, the behavior of $\left\langle v v_{z}\right\rangle /\left\langle v_{z}\right\rangle^{2}$ will always be virtually identical to the uppermost curve in Fig. 5 , since the collisional momentum loss by ions is then essentially independent of the mass ratio.

We may conclude from Figs. $2-5$ that the assumptions of a negligible pressure gradient and a weakly varying dynamical friction coefficient $\kappa(z)$ underlying the simple model (10) are valid under either (or both) of the following circumstances: (i) the ions are at least as massive as the neutrals $(m \geq M)$; (ii) charge transfer is the dominant ion-neutral-species interaction $\left(\sigma_{c} \gg \sigma_{e}\right)$.

\section{Comparison with the continuum model}

In Fig. 6 we compare the converged electric-field profile $E(z)$ from run A3 with profiles obtained by numerical in-

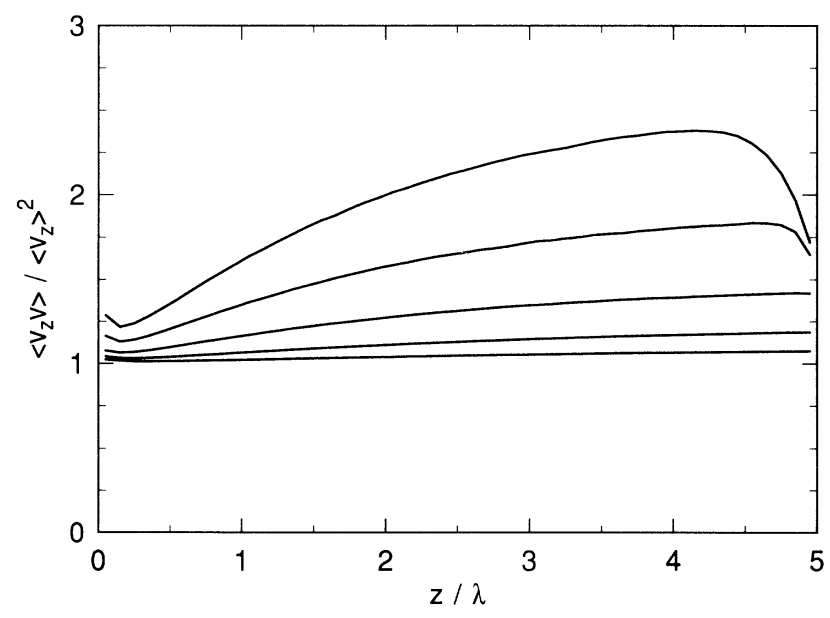

FIG. 4. Behavior of the ratio $\left\langle v v_{z}\right\rangle /\left\langle v_{z}\right\rangle^{2}$ that occurs in the dynamical friction coefficient $\kappa(z)$ defined by (55) for the case of elastic scattering with $d / \lambda=5$ and mass ratios $m / M=0.25$, $0.5,1.0,2.0$, and 4.0 (sequence A). The highest curve is for $m / M=0.25$ and the lowest for $m / M=4.0, \kappa(z)$ decreasing monotonically with $m / M$ for intermediate values.

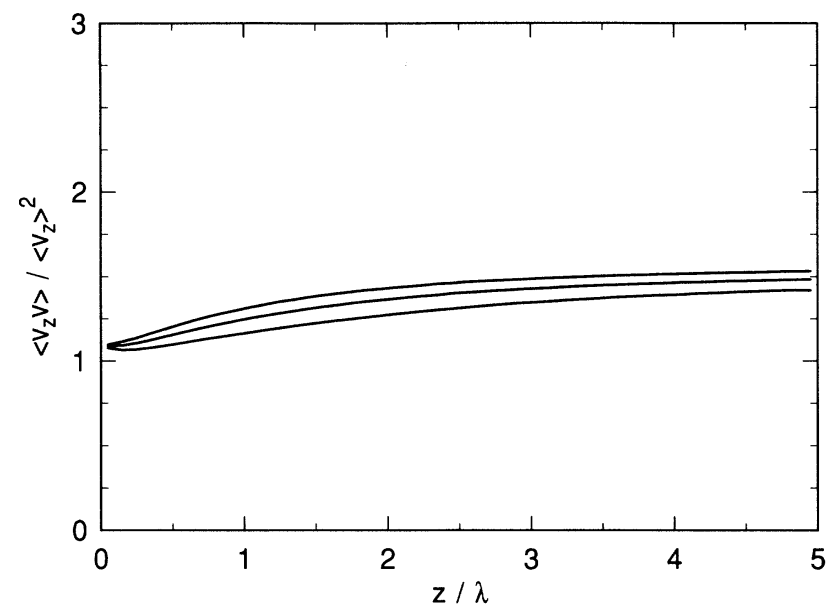

FIG. 5. The behavior of $\left\langle v v_{z}\right\rangle /\left\langle v_{z}\right\rangle^{2}$ for $m / M=1$ and cross-section ratios $\sigma_{e}: \sigma_{c}=1: 0,1: 1$, and 0:1 (sequence B). The highest curve is for $\sigma_{e}: \sigma_{c}=0: 1$ and the lowest is for $\sigma_{e}: \sigma_{c}=1: 0$.
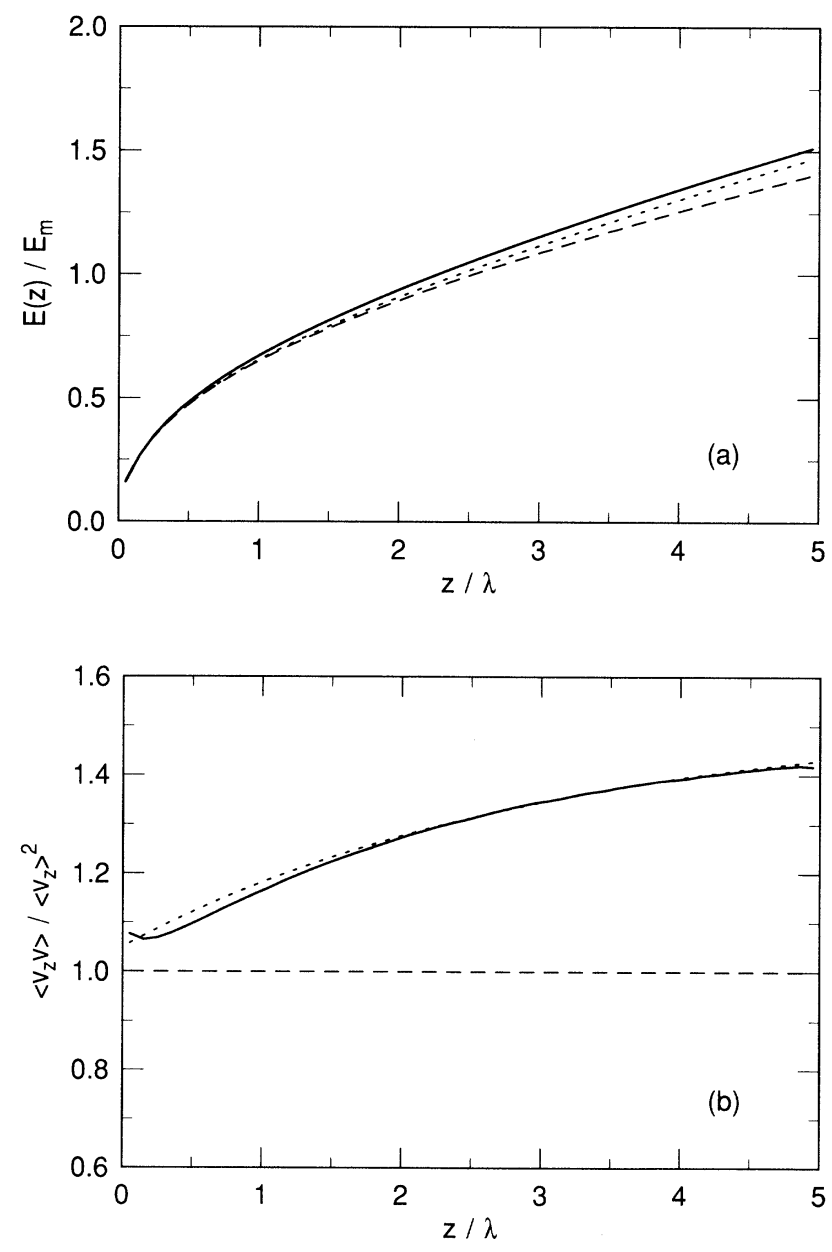

FIG. 6. (a) Comparison of the converged electric-field profile for run A3 (solid curve) with that obtained from the simple continuum model (10) with $\kappa(z)=$ constant (dashed curve) and the exponential fit (77) to $\kappa(z)$ (dotted curve). (b) The corresponding approximations to the behavior of $\left\langle v v_{z}\right\rangle /\left\langle v_{z}\right\rangle^{2}$. 
tegration of the continuum model equations (10), incorporating various simple approximations to the dynamical friction coefficient. The following functional forms were tested: (i) $\kappa(z)=$ const, corresponding to the crude assumption $\left\langle v v_{z}\right\rangle /\left\langle v_{z}\right\rangle^{2} \equiv 1$ in (55); (ii) a linear fit $\kappa(z)=\kappa_{0}+\left(\kappa_{1}-\kappa_{0}\right) z / d$, with initial and final values $\kappa_{0}$ and $\kappa_{1}$ determined empirically from the data of Fig. 4; and (iii) the exponential form

$$
\kappa(z)=\kappa_{0}+\left(\kappa_{\infty}-\kappa_{0}\right)[1-\exp (-k z / \lambda)],
$$

where the asymptotic value $\kappa_{\infty}$ is estimated from the simulation and the factor $k$ is adjusted to optimize the fit; $k=3$ in Fig. 6. [In general, expression (77) was found to give a good description of the behavior of $\kappa(z)$ when $m / M \geq 1$ or $\sigma_{c}>\sigma_{e}$, although the ad hoc choice of the parameter $\alpha$ in (75) often results in an initial perturbation from this idealized form.]

Figure 6 shows the results obtained with $\kappa(z)=$ const and the exponential fit (77); the linear approximation gives a profile for the electric field that lies between the profiles obtained in these two cases. Note that a slight discrepancy persists between the converged Monte Carlo profile for $E(z)$ and that obtained from (10), even with a relatively accurate fit for $\kappa(z)$. This may be attributable in part to the neglect of the pressure gradient in the momentum equation of the simple continuum model; it is evident from Fig. 2 that with $m / M=1$ and pure elastic scattering, this term accounts for about $5 \%$ of the field acceleration $q E / m$. Indeed, for run A5 with $m / M=4$, both the relative magnitude of the pressure gradient and the variation of $\kappa(z)$ are much less significant, and a very accurate agreement between the Monte Carlo simulations and the continuum model is observed. For mass ratios less than unity, however, we find that Eqs. (10) fail to produce a satisfactory quantitative agreement with the simulations (unless charge transfer dominates).

\section{Angular and energy distributions}

As noted in Sec. I, the angular and energy distributions of ions impinging on the cathode are of practical interest in modeling the etching of microelectronic circuit features. Our simulations facilitate a quantitative examination of the dependence of these distributions on the various sheath parameters. We shall confine ourselves here to presenting and briefly discussing some representative results; a detailed application of the incident ion distributions to etch models is given in [3].

Typical measured distributions are shown in Figs. 7 and 8 . In the former we show the angular and energy distributions of incident ions for runs A1, A3, and A5, cor-
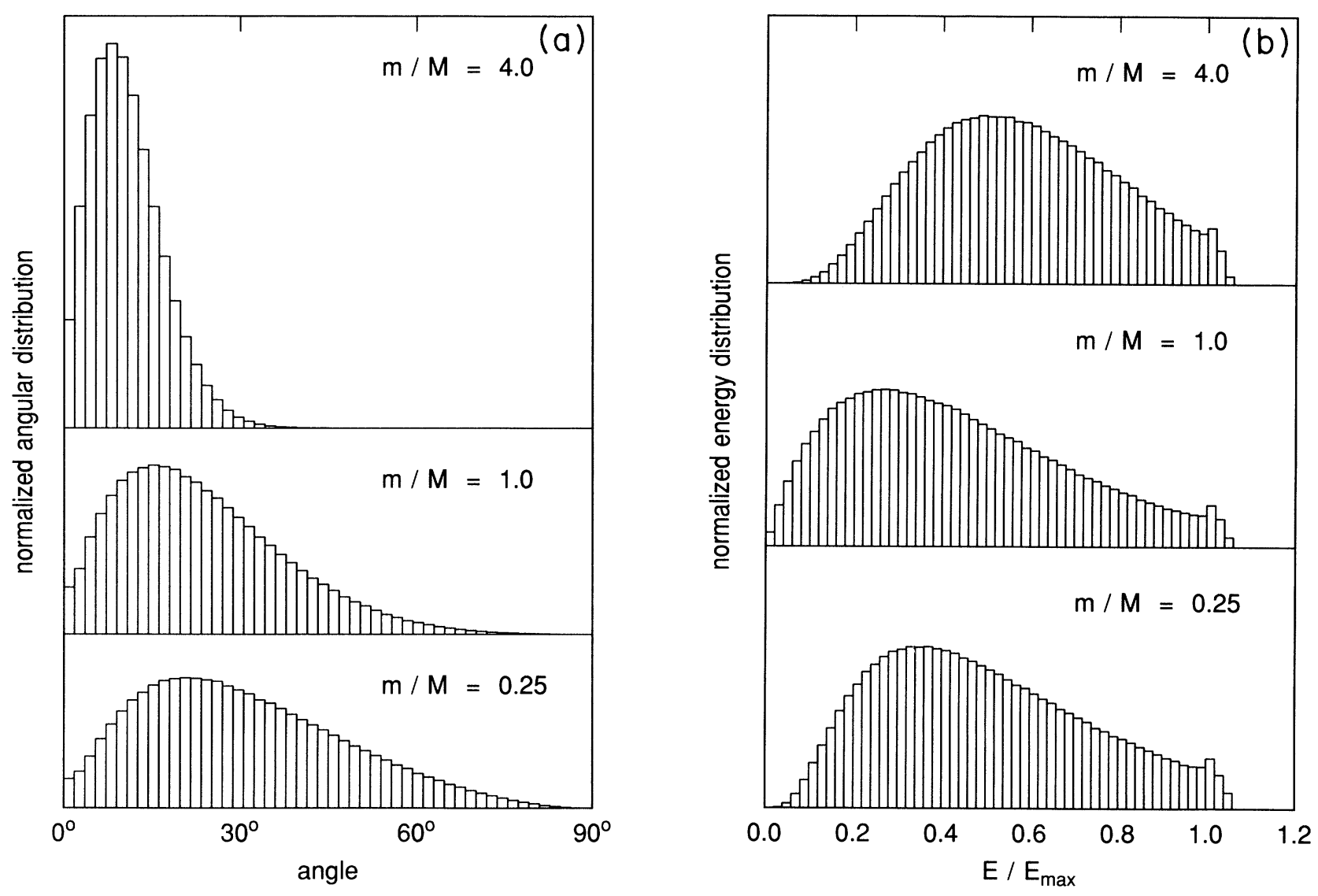

FIG. 7. Angular and energy distributions of incident ions for the case of elastic scattering with $d / \lambda=5$ and mass ratios $m / M=0.25,1.0$, and 4.0 (runs A1, A3, and A5). 
responding to pure elastic scattering with different mass ratios. In the latter, the mass ratio is fixed at unity while the ratio $\sigma_{e}: \sigma_{c}$ of cross sections is varied (sequence $\mathrm{B}$ ). In the angular distributions, the "spikes" at normal incidence for cases where $\sigma_{c} \neq 0$ correspond to ions whose final encounter with a neutral species is a charge-transfer event (these spikes have been truncated in Fig. 8).

Note that with $d / \lambda=5$, there is still a discernible fraction of ions that traverse the sheath without encountering neutral species. These are apparent as a slight excess in the angular distribution at $\theta=0$, and in the energy distribution at $\varepsilon=\varepsilon_{\max }$, the kinetic energy corresponding to the sheath potential [the Maxwellian component of the initial distribution (75) induces a "smearing" of this effect in Figs. 7 and 8, and accounts for those ions with energies slightly greater than $\varepsilon_{\max }$.

It is interesting to examine the relative contributions of scattered and unscattered ions to the angular and energy distributions as $d / \lambda$ is increased. Figure 9 shows the case of elastic scattering (sequence $\mathrm{C}$ ); analogous results hold for charge transfer.

When charge transfer dominates, all ions impinge on the cathode with nearly normal incidence when the parameter $\alpha$ is much less than unity. In that case, it is possible to derive an analytic expression for the energy dis-

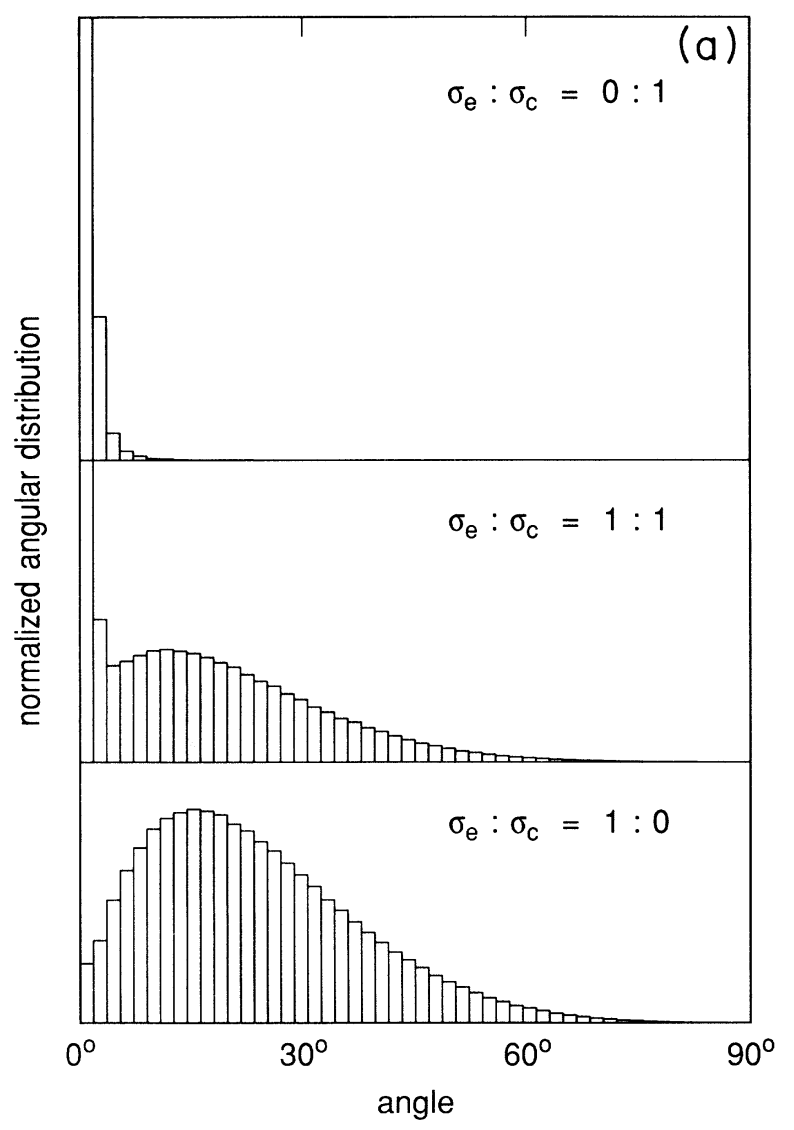

tribution [40] that reduces to an exponential decline with $\varepsilon / \varepsilon_{\max }$ in the case $d / \lambda \gg 1$. The distributions obtained when $\sigma_{e}: \sigma_{c}=0: 1$ (for $d / \lambda=5$ shown in Fig. 8, and for other $d / \lambda$ values not shown) are in good agreement with experimental results [40].

When elastic scattering dominates the ion-neutralspecies cross section, it is also possible to derive closedform expressions for the angular and energy distributions of those ions that actually suffer a scattering event in sheaths of low collisionality $(d / \lambda<<1)$ directly from the Boltzmann equation. This derivation, and a comparison with results from the Monte Carlo program, may be found in [41].

Note that although the incident ion distributions shown here are all normalized to unit area, they should not be interpreted as probability density functions for individual ions to arrive at a given angle or energy. Since the Monte Carlo simulations model a steady state, the totality of ions that are followed sequentially in the program must be interpreted as those that traverse the sheath in a fixed time interval. For example, with $10^{6}$ ions, Figs. 7-9 would represent the ions received at the cathode in an interval $\Delta t=10^{6} q / J A$, where $A$ is the cathode area and $J / q$ is the (constant) ion flux in the sheath. Thus the distributions obtained by a simple accu-

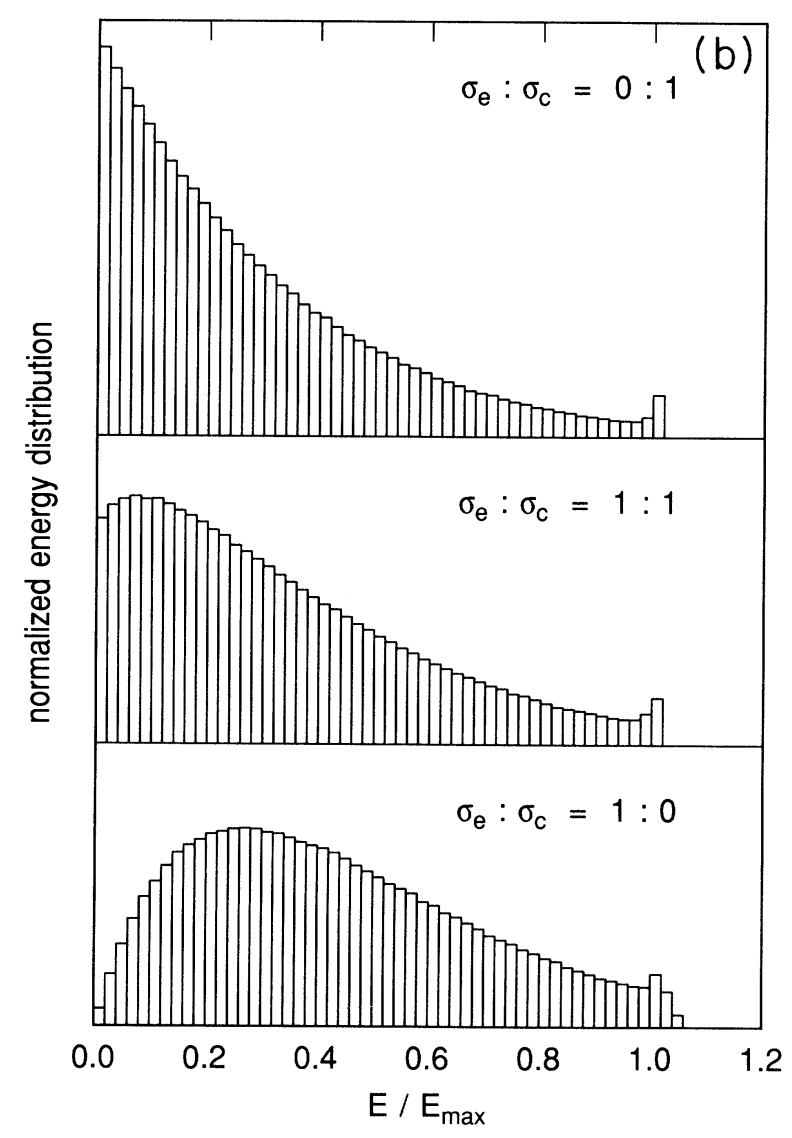

FIG. 8. Angular and energy distributions of incident ions for $m / M=1$ and cross-section ratios $\sigma_{e}: \sigma_{c}=1: 0,1: 1$, and $0: 1$ (sequence B). 
mulation of ions in discrete angle and energy bins as they arrive at the cathode are proportional to the ion fluxes per unit angle $F(\theta)$ and per unit energy $F(\varepsilon)$.

In terms of the ion distribution function $f(v, \theta, d)$ at $z=d$, the total ion flux $F$ at the cathode is given by

$$
F=\iint v f(v, \theta, d) 2 \pi \sin \theta d \theta v^{2} d v .
$$

The corresponding flux per unit angle may be expressed as

$$
F(\theta)=2 \pi \sin \theta \int f(v, \theta, d) v^{3} d v
$$

while the flux per unit energy is obtained by making the substitution $v=\sqrt{2 \varepsilon / m}$,

$$
F(\varepsilon)=\frac{2 \varepsilon}{m^{2}} \int f(\sqrt{2 \varepsilon / m}, \theta, d) 2 \pi \sin \theta d \theta .
$$

Thus the distributions shown in Figs. 7-9 must be regarded as representing the quantities (79) and (80). Further discussion of these matters may be found in [3].

It is not difficult to determine also the bivariate distribution $F(\theta, \varepsilon)$ of ions with respect to incidence angle and kinetic energy from the simulations, although large numbers are required to reduce sampling noise to an acceptable level (see [2]). We omit these results for the sake of

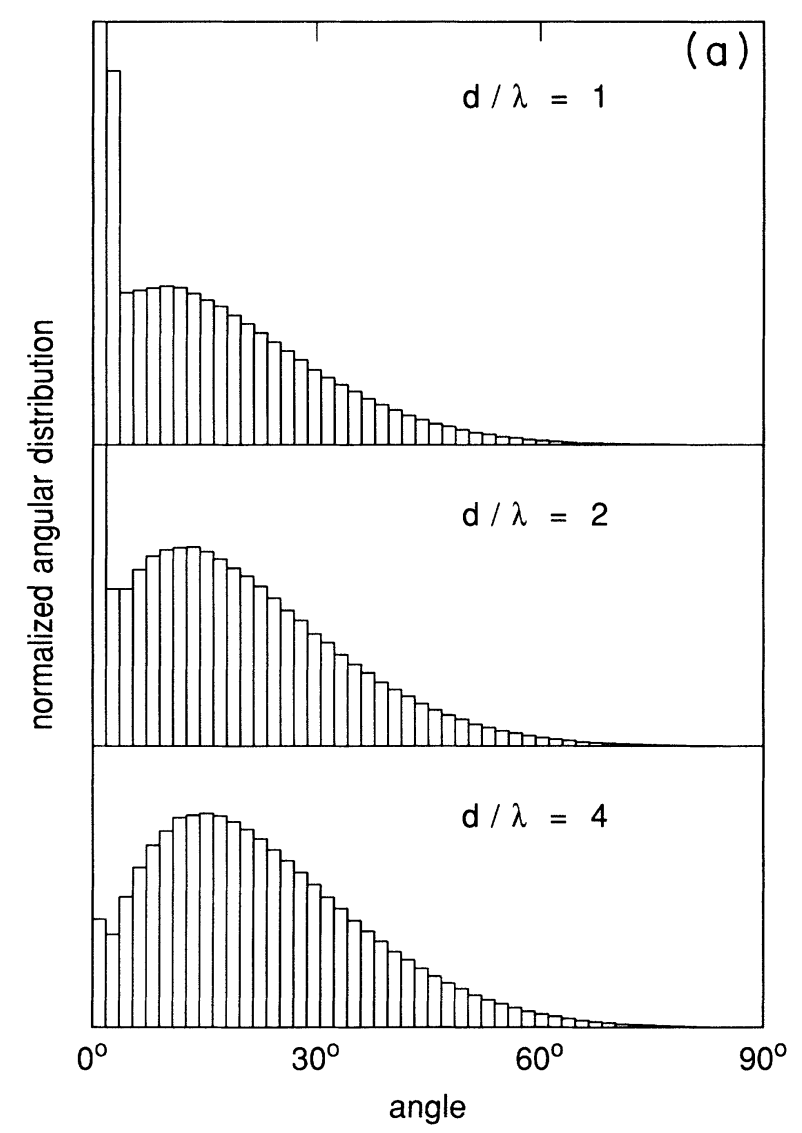

brevity.

Finally, it should be emphasized that the distributions shown in Figs. 7-9 arise from the very simple forms assumed for the ion-neutral-species interaction, and might be significantly altered by the use of more realistic collision models (energy-dependent cross sections, etc.). Liu, Huppert, and Sawin [42] give a detailed comparison of ion distributions obtained from experimental measurements and from simulations similar to those presented herein; they ascribe observed discrepancies to the idealized separation of the cross section into distinct elasticscattering and charge-transfer components.

\section{CONCLUDING REMARKS}

The structure of collisional plasma sheaths is governed by a system of coupled integro-differential equations for the ion distribution function $f(\mathbf{v}, z)$ and the selfconsistent electric field $E(z)$ - see Eqs. (14) - (17) — and in general is not amenable to straightforward analytical treatment. Our Monte Carlo simulations identify regimes under which a much simpler and thus more tractable model of the form (10), formulated in terms of intuitive macroscopic quantities, can yield substantively accurate descriptions of the sheath structure.

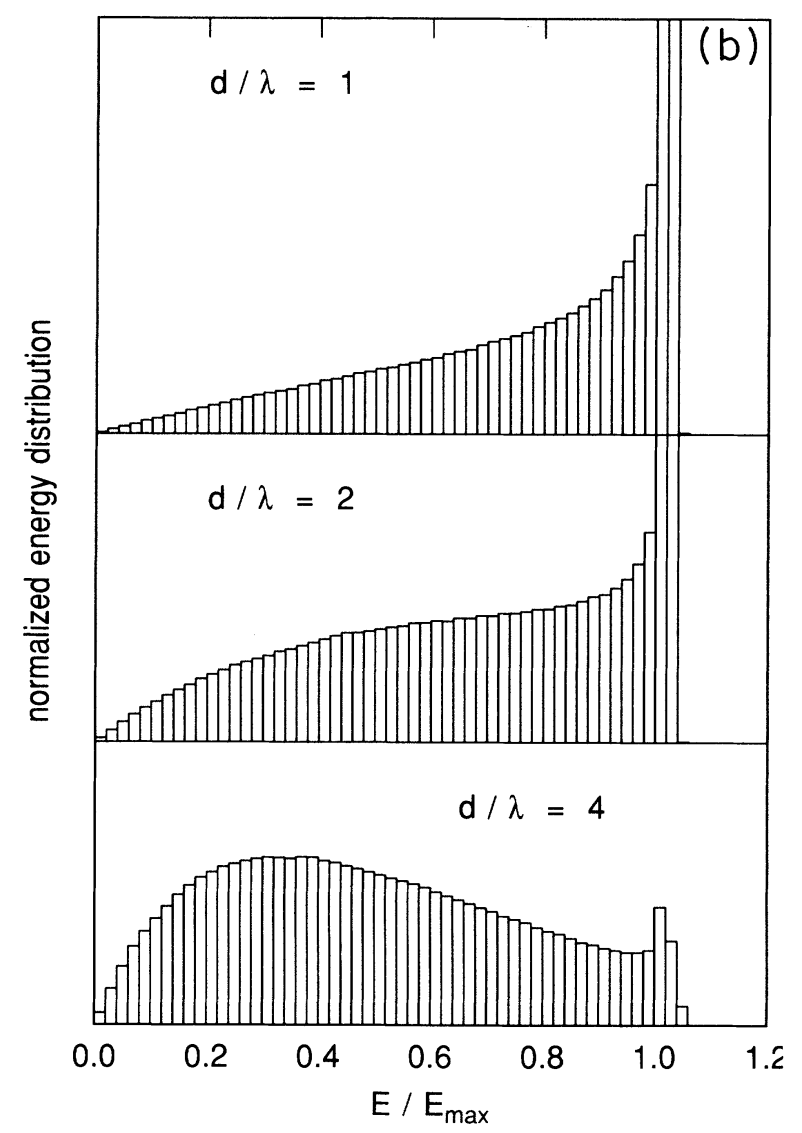

FIG. 9. Angular and energy distributions of incident ions for the case of elastic scattering with mass ratio $m / M=1.0$ and $d / \lambda=1$, 2 , and 4 (sequence $\mathrm{C}$ ). 
Specifically, the simple continuum model is appropriate when charge transfer dominates the ion-neutral species collision cross section or, if elastic scattering is important, when the ion-to-neutral-species mass ratio is not less than unity. For elastic scattering with mass ratios less than unity, a credible continuum model will probably require higher moments of the ion distribution.
Furthermore, the strong perturbation of the distribution function observed in the simulations near the cathode (assumed to absorb all ions incident on it) suggests that in such cases it is unsatisfactory to treat the sheath structure purely as an initial-value problem as discussed in Sec. II.
[1] C. B. Zarowin, J. Electrochem. Soc. 130, 1144 (1983); J. Vac. Sci. Technol. A 2, 1537 (1984).

[2] J. I. Ulacia F. and J. P. McVittie, J. Appl. Phys. 65, 1484 (1989).

[3] M. Dalvie, R. T. Farouki, and S. Hamaguchi, IEEE Trans. Electron. Devices (to be published).

[4] M. J. Kushner, J. Appl. Phys. 58, 4024 (1985).

[5] B. E. Thompson, H. H. Sawin, and D. A. Fisher, J. Appl. Phys. 63, 2241 (1988).

[6] W. B. Pennebaker, IBM J. Res. Dev. 23, 16 (197\%,

[7] D. J. Economou, D. R. Evans, and R. C. Alkire, J. Electrochem. Soc. 135, 756 (1988).

[8] Inelastic events (excitation, ionization, dissociation) and energy-dependent cross sections are not difficult in principle to incorporate in the Monte Carlo method, but are omitted here in order to keep the dynamics simple.

[9] M. A. Lieberman, IEEE Trans. Plasma Sci. PS-16, 638 (1988); PS-17, 338 (1989).

[10] S. A. Self, Phys. Fluids 6, 1762 (1963).

[11] D. B. Graves and K. F. Jensen, IEEE Trans. Plasma Sci. PS-14, 78 (1986).

[12] J.-P. Boeuf, J. Appl. Phys. 63, 1342 (1988).

[13] M. Surendra, D. B. Graves, and G. M. Jellum, Phys. Rev. A 41, 1112 (1990).

[14] D. A. Doughty, E. A. Den Hartog, and J. E. Lawler, Phys. Rev. Lett. 58, 2668 (1987); E.A. Den Hartog, D.A. Doughty, and J. E. Lawler, Phys. Rev. A 38, 2471 (1988).

[15] T. J. Sommerer, J. E. Lawler, and W. N. G. Hitchon, J. Appl. Phys. 64, 1775 (1988); T. J. Sommerer, W. N. G. Hitchon, and J. E. Lawler, Phys. Rev. A 39, 6356 (1989).

[16] The electron density $n_{e}$ diminishes rapidly in the presheath region; since it influences the sheath structure only through its contribution to the right-hand side of Poisson's equation, we define the presheath-sheath interface to be the point where $n_{e}$ has dropped to some small fraction of the ion density.

[17] B. Chapman, Glow Discharge Processes (Wiley, New York, 1980).

[18] These boundary conditions are more appropriate to the original context of the Child-Langmuir law - thermionic emission of electrons from a heated filament [C.D. Child, Phys. Rev. 32, 492 (1911); I. Langmuir, Phys. Rev. 2, 450 (1913)] — than to plasma sheaths.

[19] R. T. Farouki, M. Dalvie, and L. F. Pavarino, J. Appl. Phys. 68, 6106 (1990).

[20] D. Bohm, in The Characteristics of Electrical Discharges in Magnetic Fields, edited by A. Guthrie and R. K. Wakerling (McGraw-Hill, New York, 1949), p. 77.

[21] G. H. Wannier, Bell Syst. Tech. J. 32, 170 (1953).

[22] E. W. McDaniel and E. A. Mason, The Mobility and Diffusion of Ions in Gases (Wiley, New York, 1973); E. A.
Mason and E. W. McDaniel, Transport Properties of Ions in Gases (Wiley, New York, 1988).

[23] L. B. Loeb, Basic Processes of Gaseous Eelctronics (University of California Press, Berkeley, 1955).

[24] A. von Engel, Ionized Gases, (Clarendon, Oxford, 1965).

[25] R. N. Franklin, Plasma Phenomena in Gas Discharges (Clarendon, Oxford, 1976).

[26] It is more useful to consider $u$ as a function of the ratio $E / p_{g}$ of the field strength to the gas pressure, if the latter is to be varied. Note in Eq. (8) that $\kappa \propto \lambda^{-1} \propto p_{g}$ (see Sec. II below) for a fixed gas temperature.

[27] When $\kappa=$ const, Eqs. (10) may be combined into a single equation $d u / d E+\kappa \epsilon_{0} u^{2} / J=q \epsilon_{0} E / m J$. The substitution $u=(J d F / d E) / \kappa \epsilon_{0} F$ then transforms this into a linear second-order equation, $d^{2} F / d E^{2}-E F / E_{*}^{3}=0$, where $E_{*}^{3}=m J^{2} / q \kappa \epsilon_{0}^{2}$. Solutions to the latter may be expressed in the form $F(E)=\sqrt{E}\left[\alpha I_{1 / 3}(\mathscr{E})+\beta I_{-1 / 3}(\mathscr{E})\right]$, where $I_{1 / 3}$ and $I_{-1 / 3}$ are modified Bessel functions of the argument $\mathscr{E}=\frac{2}{3}\left(E / E_{*}\right)^{3 / 2}$. On choosing $\alpha$ and $\beta$ to satisfy the initial conditions and substituting back, we obtain a solution for the sheath structure giving the stream velocity $u$ and distance $z$ in terms of the local-field strength $E$. [See G. M. Murphy, Ordinary Differential Equations and Their Solutions (Van Nostrand, Princeton, 1960), pp. 20, 315, and 339.]

[28] C. Cercignani, The Boltzmann Equation and Its Applications (Springer-Verlag, New York, 1988).

[29] W. P. Allis, in Motions of Ions and Electrons, edited by S. Flügge, Handbuch der Physik Vol. 21 (Springer-Verlag, Berlin, 1956).

[30] Formally, the sheath structure can be regarded as an initial-value problem only in the limit $d / \lambda \rightarrow \infty$, since the assumption that all ions are absorbed by the cathode imposes a boundary condition on $f(\mathbf{v}, z)$ at $z=d$. When the ion-to-neutral-species mass ratio is not less than unity or charge transfer dominates, however, this boundary condition has an insignificant effect on the sheath structure, even for finite $d / \lambda$ (see the results of the simulations in Sec. IV).

[31] L. D. Landau and E. M. Lifshitz, Mechanics (Pergamon, Oxford, 1960).

[32] R. Forman, Phys. Rev. 123, 1537 (1961).

[33] J. H. Ingold, J. Appl. Phys. 40, 55 (1969); Phys. Fluids 15, 75 (1972).

[34] A. Metze, D. W. Ernie, and H. J. Oskam, Phys. Rev. A 39, 4117 (1989).

[35] J. M. Hammersley and D. C. Handscomb, Monte Carlo Methods (Wiley, New York, 1964).

[36] Expression (64) is valid for both positive and negative $v_{z}$ when $E>0$

[37] D. E. Knuth, The Art of Computer Programming, Volume 
2: Seminumerical Algorithms (Addison-Wesley, Reading, MA, 1981).

[38] However, the simulations indicate that when the ions acquire large random velocities - as in the case of small mass ratios - the presence of an absorbing wall at $z=d$ can significantly influence the sheath structure in its vicinity.
[39] J. E. Lawler, Phys. Rev. A 32, 2977 (1985).

[40] W. D. Davis and T. A. Vanderslice, Phys. Rev. 131, 219 (1963).

[41] S. Hamaguchi, R. T. Farouki, and M. Dalvie, IBM Research Report No. RC 16622 (unpublished).

[42] J. Liu, G. L. Huppert, and H. H. Sawin, J. Appl. Phys. 68, 3916 (1990). 